\title{
Optimally combined headway and timetable reliable public transport system
}

\author{
Balázs Varga ${ }^{\mathrm{a}, *}$, Tamás Tettamantia ${ }^{\mathrm{a}}$, Balázs Kulcsár ${ }^{\mathrm{b}}$ \\ ${ }^{a}$ Department of Control for Transportation and Vehicle Systems, Budapest University of \\ Technology and Economics, Stoczek J. u. 2, H-1111, Budapest, Hungary \\ ${ }^{b}$ Department of Electrical Engineering, Chalmers University of Technology, Hörsalsvägen \\ 9-11, SE-412-96, Gothenburg, Sweden
}

\begin{abstract}
This paper presents a model-based multiobjective control strategy to reduce bus bunching and hence improve public transport reliability. Our goal is twofold. First, we define a proper model, consisting of multiple static and dynamic components. Bus-following model captures the longitudinal dynamics taking into account the interaction with the surrounding traffic. Furthermore, bus stop operations are modeled to estimate dwell time. Second, a shrinking horizon model predictive controller (MPC) is proposed for solving bus bunching problems. The model is able to predict short time-space behavior of public transport buses enabling constrained, finite horizon, optimal control solution to ensure homogeneity of service both in time and space. In this line, the goal with the selected rolling horizon control scheme is to choose a proper velocity profile for the public transport bus such that it keeps both timetable schedule and a desired headway from the bus in front of it (leading bus). The control strategy predicts the arrival time at a bus stop using a passenger arrival and dwell time model. In this vein, the receding horizon model predictive controller calculates an optimal velocity profile based on its current position and desired arrival time. Four different weighting strategies are proposed to test (i) timetable only, (ii) headway only, (iii) balanced timetable - headway tracking and (iv) adaptive
\end{abstract}

\footnotetext{
* Corresponding author

Email addresses: varga.balazs@mail.bme.hu (Balázs Varga), tettamanti@mail.bme.hu (Tamás Tettamanti), kulcsar@chalmers.se (Balázs Kulcsár)
}

Preprint submitted to Transportation Research Part C: Emerging TechnologiesApril 14, 2018 
control with varying weights. The controller is tested in a high fidelity traffic simulator with realistic scenarios. The behavior of the system is analyzed by considering extreme disturbances. Finally, the existence of a Pareto front between these two objectives is also demonstrated.

Keywords: Bus bunching, MPC control, Autonomous vehicles, Multiobjective optimization, Timetable reliability

\section{Introduction}

In populated urban areas, often in peak hours, public transport service providers are unable to ensure a temporally and spatially homogeneous service. Increased passenger demand and interactions with dense traffic are contributing 5 factors to bus bunching. At frequent lanes, if the schedule cannot be held and a bus arrives at the stop late, number of passengers is winding up. Increased dwell times further delay the bus. The headway between the current and the successor bus will eventually decrease so much that buses stick together. This instability in public transport is called bus bunching (Pilachowski, 2009). It leads to non-homogeneous utilization of buses and therefore degradation of service quality. Furthermore, passengers tend to board the first bus to reduce their own travel delay.

Bus bunching was first described in (Newell and Potts, 1964). Through improvements in sensor technology (GPS, Automatic Vehicle Location (AVL),

15 Automatic Passenger Count (APC)) the phenomenon could be better grasped and it opened ways to deal with this problem. (Mandelzys and Hellinga, 2010) employed AVL and APC methods to identify bottlenecks at urban bus routes. (Fonzone et al., 2015) studied the effect of passenger arrival patterns on bunching, concluding that unexpected passenger demands are the root cause of bunch-

20 ing. Due to bunching the periodicity of arrivals fail and homogeneous service fannot be provided (Ap. Sorratini et al. 2008). In (Daganzo, 2009) and (Daganzo and Pilachowski, 2011) algorithms are developed to control the headway of consecutive buses. (Ampountolas and Kring, 2015) proposed cooperative 
control of buses to mitigate bunching. (Bartholdi and Eisenstein, 2012) formulated a self controlling algorithm without timetable. The above works focus solely on headway keeping, not considering adhering to the schedule. In (Xuan et al. 2011) optimal control algorithms are considered, taking into account both headway and timetable keeping. (Andres and Nair, 2017) used predictive algorithms to improve public transport reliability. Recent paper from (Yu et al.

30 2016) employ already existing information to predict bus bunching employing information from transit smart cards.

In addition to bunching, timetable reliability is and travel time prediction are two intensively researched topics. (Rahman et al., 2018) provides a predictive method based on GPS position and timetable data. A common method in improving timetable reliability provides priority to buses at signalized intersections (Estrada et al., 2016). In (Estrada et al., 2016) a velocity control method considering bus-to-bus communication and green time extension is formulated. Public transport reliability is addressed in (Nesheli et al., 2015) with bus holding, stop skipping to minimize passenger waiting time. (Jiang et al., 2017) proposed a heuristic algorithm with stop skipping or inclusion for congested high-speed train lines.

References (Fonzone et al., 2015) - (Jiang et al., 2017) seek to remedy bunching by including slack times or stop skipping. In densely populated urban areas where city space is scarce, including slack times might not be possible due to bus stop configurations (Cats et al., 2012). Furthermore, slacks are an unproductive allocation of time of time in the cycle time of buses and results in queuing at stops ((Daganzo, 2009)). Slack times can be dynamically addressed via changing the speed of the vehicle rather than holding it. In that sense, we propose a smoothed and pro-active way of slack time reduction foreseeing the trajectories 50 (headway, timetable) to track. Our method is based on a dynamic prediction to better model the vehicle's future dynamics instead of regarding the trip times between stops as random variables as done in (Xuan et al. 2011).

In this paper we present a velociy control algorithm based on communication between public transport buses and their infrastructure. The velocity control 
can act as an assistance to the driver or with the emergence of autonomous चehicles, a strict reference speed in a cruise control application Daganzo and Pilachowski, 2011). We describe an optimal, decentralized, shrinking horizon model predictive control (MPC) algorithm to achieve headway homogeneity in both time and space on an urban bus route. Several of the aforementioned works

60 consider forward-backward-headway control e.g. (Daganzo, 2009, Ampountolas and Kring, 2015, Andres and Nair, 2017). In our model predictive approach, considering the bus behind is not possible, future trajectory of the following would be an unreliable reference.

The proposed control oriented model on top of the longitudinal bus dynamics, takes into account uncertainties such as varying dwell times and delays due to interaction with traffic. The MPC is an adequate choice for predicting arrival times and calculating an optimal velocity profile. Decentralized control means there is a speed controller running on each bus. The control design is based on a quadratic cost function, weighting delays or early arrivals and deviation from the defined headway. The linear nature of the control oriented model and the constraints represented by linear relationships enables us to solve the optimization effectively on individual vehicles.

The paper is organized as follows. Methodological overview section gives an overview of the proposed system architecture and control strategy. In the 75 System modeling part the subsystems proposed in the system architecture are detailed. A passenger arrival model and a dwell time model are presented to describe operations at a bus stop. Then, a control oriented bus following model is formulated. In the Reference speed control design section shrinking horizon model predictive controllers are proposed with different weighting strategies. For comparative analysis of the controllers a simulation scenario is created in a high fidelity traffic simulator based on real world data. Next, simulation results are analyzed. First, the operation of the controller is shown for one bus, then it is extended for several buses. Finally, the system is evaluated under extreme disturbances. 


\section{Methodological overview}

Buses operate on a given route based on their timetable. During operation, due to irregular dwell time, they tend to get out of sync with the schedule and start bunching.

The goal of the control algorithm is to calculate an optimal velocity profile for each bus, which ensures its timetable and headway reliability. To this end, a model is proposed to describe bus operations on a line. This model has modular layout and can be disassembled into subsystems: the passenger arrival model and dwell time model describes operations at a bus stop. Movement between stops is characterized by the vehicle dynamics subsystem, which consists of a longitudinal car following model. Surrounding traffic conditions are also taken into consideration.

The velocity controller calculates a reference velocity profile $v_{\text {des }}$ for the bus based on two reference signals: (i) given the estimated dwell time and the scheduled departure time from a stop, a desired trajectory is calculated $x_{d e s}(t)$; (ii) to keep equidistant headways, trajectory of the leading bus $x_{r e f}(t)$ is also taken into account. By means of balancing between these conflicting references, an optimal velocity profile is formulated. The proposed system can be classified as an overlapped, decentralized control. The controlled bus only requires the historical position of the leader bus and the schedule (stop locations and desired departure times), see Figure 1. In case either of them is missing or disabled, the system can operate in either headway tracking or timetable tracking mode. The control algorithm is generic, it can be applied to different routes, fleet configurations, schedules etc.

Place Figure 1 about here.

110

Figure 2 depicts the modularity of the proposed control system. Subsystems and notations are further detailed in the following parts.

Place Figure 2 about here. 


\section{Bus stop operations}

115

This section presents the static models used for the bus bunching control algorithm. The passenger arrival model in conjunction with the dwell time model describe the operations taking place at a bus stop. The control algorithm is deterministic, thus mean values are used for dwell time prediction. The predicted dwell time is used to estimate desired arrival times. The stochastic nature of the aforementioned models is exploited in the simulation scenarios, bringing additional disturbances to the system. The scheduler is responsible for generating the reference signals for the buses.

\subsection{Passenger arrival model}

The average passenger waiting time at a stop can simply be described as half of the arrival rate of the public transport vehicle. This assumption holds if the following conditions are fulfilled: passengers arrive randomly; passengers fan get on the first arriving vehicle and vehicles have equal headways Holroyd and Scraggs, 1966). In routes with frequent service (headways with 10 minutes or less) passengers typically do not consult schedules before arriving at their stops, their arrival rate can be thought random (Dessouky et al., 2003).

In (Jolliffe and Hutchinson, 1975) three types of passengers are categorized.

a) There are passengers whose arrival time is coincident with the bus. These are the passengers who run to the stop because they see the bus coming, and thus wait zero time.

b) There are some passengers who plan their arrival at the bus stop so as to be there just before the bus comes, minimizing their expected waiting time. This decreases the average waiting time (O'Flaherty and Mangan. 1970).

c) Finally, there are completely random passenger arrivals.

140 The arrival rate of group $b$ ) and $c$ ) is described by Poisson distribution and their ratio depends on the length of bus headways. 
The time between successive arrivals is exponentially distributed with parameter $\lambda$ (arrival rate) and independent of the past. In the sequel, we use a Poisson distribution to inject passengers. However, we assume the hourly passenger demand at each stop $N_{\text {pass }}$ is known from the service provider. This demand can be split into the three aforementioned categories. Figure 3 depicts the arrival rate of each passenger type between successive bus departures $\left(t_{d e p, i}\right.$ and $\left.t_{d e p, i+1}\right)$. The area under the graph equals the number of passengers waiting at the stop. This is the number of boarding passengers $P_{b}$ which is used in the dwell time model.

Place Figure 3 about here.

\subsection{Dwell time model}

Dwell time is the average amount of time a public transport bus is stopped at the curb to serve passenger movements, including the time required to open and close the doors (Kittelson et al., 2003). Uncertainty in dwell times is a major factor for bus bunching and it directly affects travel time and service quality. The total time spent at stops can consume up to $26 \%$ of the total travel time (Rajbhandari et al. 2003). It is influenced by several factors: the configuration and occupancy of the bus, the number of boarding and alighting passengers, the configuration of stops, and the method of fare collection (e.g. on board or pre-ticketing).

According to (Kittelson et al. 2003) time spent at a bus stop can be estimated knowing the number of boarding and alighting passengers, door configuration and other factors such as low floor, fare paying method etc. The average dwell time $t_{d}$ at a bus stop can be calculated as:

$$
t_{d}=P_{b} \cdot t_{b}+P_{a} \cdot t_{a}+t_{o c},
$$

where

$t_{b}$ is the average boarding time per passenger,

$t_{a}$ is the average alighting time per passenger,

${ }_{165} t_{o c}$ is the door opening and closing time, 
$P_{b}$ is the number of boarding passengers and

$P_{a}$ is the number of alighting passengers.

The boarding $t_{b}$ and alighting time $t_{a}$ of individual passengers can be modeled with normally distributed random processes. In the control design, average values are used: $t_{o c}=3.5 \mathrm{~s}, t_{a}=1.2 \mathrm{~s}$ and $t_{b}=1.5 \mathrm{~s}$, the dwell time is proportional to the number of boarding and alighting passengers (Kittelson et al. 2003). $P_{b}$ is obtained from the passenger arrival model (Section 3.1), the number of alighting passengers at each stop $P_{a}$ is considered to be known from the service provider.

\subsection{Scheduler}

Buses operate on a route based on a timetable. The scheduler, as a supervisor, defines where a bus shall be at a given time based on its timetable $x_{d e s}(t)$. Additionally, the reference position based on the leading bus $x_{r e f}(t)$ is calculated here. The timetable is static and obtained from the transport service provider in minutes resolution. For control design purposes this timetable was refined to seconds.

\section{Vehicle dynamics}

The discrete-time model for the bus dynamics is based on the Optimal Velocity Model (OVM) (Bando et al., 1995). Position $x(k)$, velocity $v(k)$ and acceleration $a(k)$ of a vehicle can be given as follows:

$$
\begin{gathered}
x(k+1)=x(k)+v(k) \Delta t, \\
v(k+1)=v(k)+a(k) \Delta t, \\
a(k)=\frac{1}{\tau}\left(v_{\text {des }}(k)-v(k)\right)
\end{gathered}
$$

where position $x(k+1)$ and velocity $v(k+1)$ denote the states over the time period of $[k \Delta t,(k+1) \Delta t]$ with discrete time step index $k$ and sampling time $\Delta t$. $v_{\text {des }}(k)$ is the desired velocity at time step $k . \tau$ is a model parameter capturing the sensitivity of drivers to the change of their desired velocity. According 
to (Helbing and Tilch, 1998) it shall be calibrated between $1.25 \mathrm{~s}$ and $2.5 \mathrm{~s}$. Too small values would result in rapid acceleration or deceleration towards the desired velocity. With autonomous vehicles these parameters could change, but still it is preferred to mimic the behavior of human drivers so their presence does not perturb traffic significantly and does not disturb other drivers participating in traffic (Kesting et al., 2008).

The above equations can be written into state space form with $v_{\text {des }}(k)$ being the controlled variable of the system: it serves as a display to the driver or a strict reference in case of autonomous driving. $X(k)=[v(k), x(k)]^{T}$ is the vector of system states at time step $k$. The state space representation of the system is therefore:

$$
\left[\begin{array}{l}
v(k+1) \\
x(k+1)
\end{array}\right]=\left[\begin{array}{cc}
1-\frac{\Delta t}{\tau} & 0 \\
\Delta t & 1
\end{array}\right]\left[\begin{array}{l}
v(k) \\
x(k)
\end{array}\right]+\left[\begin{array}{c}
\frac{\Delta t}{\tau} \\
0
\end{array}\right] v_{\text {des }}(k) .
$$

\subsection{Traffic disturbance}

An additive error structure is proposed to include the adverse effect of other vehicles participating in traffic. The control input $v_{\text {des }}(k)$ is reduced as the average velocity decreases or traffic density increases on a link. Thus the velocity equation becomes:

$$
v(k+1)=v(k)+\frac{\Delta t}{\tau}\left(v_{\text {des }}(k)-v(k)-v_{\text {dist }}\left(\rho_{i}, k\right)\right),
$$

where $v_{\text {dist }}\left(\rho_{i}, k\right)$ is the velocity disturbance which is function of the traffic density $\rho_{i}$ on link $i$ at time step $k$. The disturbance is included as a relaxation term with relaxation parameter $\beta \in[0,1]$ :

$$
v_{\text {dist }}\left(\rho_{i}, k\right)=\beta\left(v_{\text {des }}(k)-v_{\text {fund }}\left(\rho_{i}, k\right)\right) .
$$

$\beta$ describes relaxation of bus speed towards a traffic dependent equilibrium velocity. With this term, road link specific obstacles such as traffic lights or bottlenecks can be considered. The smaller $\beta$ is the slower vehicles adjust their velocity to the macroscopic velocity (Hoogendoorn and Bovy, 2001), (Van den 
Berg et al. 2003). In Equation (7) $v_{\text {fund }}\left(\rho_{i}, k\right)$ denotes the macroscopic equilibrium velocity (Daganzo and Geroliminis, 2008) at link $i$ and time step $k$. In the followings, for the sake of simplicity the argument $\rho_{i}$ will be omitted from $v_{\text {fund }}(k)$. Note that if the desired velocity falls below the equilibrium speed $\left(v_{\text {des }}(k)<v_{\text {fund }}(k)\right)$ the sign of the disturbance term changes. It forces the vehicle to increase its speed, in order not to delay traffic.

Substituting (7) into (6) results in the following velocity equation:

$$
v(k+1)=v(k)+\frac{\Delta t}{\tau}\left(v_{\text {des }}(k) \cdot(1-\beta)-v(k)+\beta \cdot v_{\text {fund }}(k)\right) .
$$

If the bus travels on dedicated bus lane the disturbance term can be omitted by selecting $\beta=0$ or very small in Equation (8).

\section{Reference speed control design}

In this section the controller design process is outlined. First, the bus following model is augmented with two position references which shall be tracked by the controller. Then, the formulation of the optimization problem and the MPC design is presented. Next, different weighting strategies are proposed to enhance timetable or headway reliability of buses. In addition, the Pareto Front of the two control objectives is formulated. Finally, three additional control strategies are outlined as benchmarks to the proposed controllers.

\subsection{Reference tracking}

To ensure public transport service homogeneity, we propose a reference tracking controller. Set points are designed to increase public transport reliability both in time (schedule) and space (headway). The control algorithm shall accomplish two objectives: timetable tracking and reduction of bus bunching. To this end, two error terms are introduced: $z_{1}$ and $z_{2} . z_{1}(k)=x_{\text {des }}(k)-x(k)$ is the difference between $x_{\text {des }}(k)$ (which is a reference position based on the timetable and the estimated dwell time) and the actual position of the bus. The second error term $z_{2}(k)=x_{r e f}(k)-x(k)$ denotes headway tracking (i.e. reducing 
bunching). $z_{2}$ is the difference between the actual position of the controlled vehicle and the shifted position of the leading bus. To minimize bunching, the trajectory of the leading bus is shifted by one headway and used as a reference to the controlled vehicle, see Figure 4 .

Place Figure 4 about here.

If the bus follows this trajectory, one headway distance is guaranteed in an insensitive way to the actual velocity of the leading bus. If the actual headway between the two buses is larger than the prediction horizon, the reference trajectory $x_{r e f}(k)$ is known for every time iteration. (The leading bus has already traveled on that trajectory so this information exists.) The state space representation of the system with the two performance outputs (reference trajectories) is as follows:

$$
\begin{aligned}
& \overbrace{\left[\begin{array}{l}
v(k+1) \\
x(k+1)
\end{array}\right]}^{X(k+1)}=\overbrace{\left[\begin{array}{cc}
1-\frac{\Delta t}{\tau} & 0 \\
\Delta t & 1
\end{array}\right]}^{A} \overbrace{\left[\begin{array}{l}
v(k) \\
x(k)
\end{array}\right]}^{X(k)}+\overbrace{\left[\begin{array}{c}
\frac{\Delta t}{\tau}(1-\beta) \\
0
\end{array}\right]}^{B_{u}} \overbrace{v_{\text {des }}(k)}^{u(k)}+\overbrace{\left[\begin{array}{ccc}
\frac{\Delta t}{\tau} \beta & 0 & 0 \\
0 & 0 & 0
\end{array}\right]\left[\begin{array}{c}
v_{\text {fund }}(k) \\
x_{\text {des }}(k) \\
x_{\text {ref }}(k)
\end{array}\right]}^{B_{\varsigma}} \\
& \overbrace{\left[\begin{array}{l}
z_{1}(k) \\
z_{2}(k)
\end{array}\right]}^{z(k)}=\overbrace{\left[\begin{array}{rr}
0 & -1 \\
0 & -1
\end{array}\right]}^{C} \overbrace{\left[\begin{array}{l}
v(k) \\
x(k)
\end{array}\right]}^{X(k)}+\overbrace{\left[\begin{array}{lll}
0 & 1 & 0 \\
0 & 0 & 1
\end{array}\right]}^{D} \overbrace{\left[\begin{array}{c}
v_{\text {fund }}(k) \\
x_{\text {des }}(k) \\
x_{\text {ref }}(k)
\end{array}\right]}^{\varsigma(k)}
\end{aligned}
$$

\subsection{Shrinking horizon optimal control solution}

The control oriented model is used as basis of a shrinking horizon MPC design (Maciejowski, 2002). The goal of the controller is calculating an optimal velocity profile between the actual position of the vehicle and the next stop, while taking into account several uncertainties, such as the adverse effect of traffic and randomness of dwell time. It is crucial to have accurate prediction for the dwell time and average traffic velocity. The desired arrival time $t_{E T A}$ is the scheduled departure time $t_{E T D}$ from the stop minus the modeled dwell time $t_{d}$ (Section 3.1 and 3.2). (ETA and ETD stand for estimated time of arrival 
and departure, respectively.)

$$
t_{E T A}=t_{E T D}-t_{d}
$$

A shrinking horizon strategy is chosen, where the horizon length is the estimated travel time to the next stop, see Figure 5 . The interval between the actual $t_{0}$ and the $t_{E T A}$ is split into $\mathrm{N}$ equidistant time samples to run the prediction model and perform the optimization. The initial horizon length of the controller $N_{0}$ 235 is calculated upon the bus departs from a stop: $N_{0}=\frac{t_{E T A}-t_{0}}{\Delta t}$. In every time step the prediction horizon decreases by one. By the last time step the bus shall arrive the desired stop. To avoid small or even negative horizon lengths (due to lateness or being close to the stop) a lower boundary for the horizon length is defined: $N=\max \left\{N, N_{\min }\right\}$, where $N_{\min }=5$. Every subsystem in the model is discrete with the same sampling time, $\Delta t=1 \mathrm{~s}$.

Place Figure 5 about here.

Consider the state space representation in Equation (9) and tracking performance Equation (10) and extend it for $N$ horizon, see Equation (12). The system state $X(k)$ is measured at time step $k$. Then, for a finite horizon length $N$ the future states $X(k+i \mid k)$ are calculated along with the corresponding control inputs $u(k+i-1 \mid k)$ and uncontrolled external input signals $\varsigma(k+i-1 \mid k)$ . Predicted state is denoted as $X(k+i \mid k)$, where time step $k$ at the right side within the parentheses denotes the current time, and $k$ at the left side the prediction step with running index $i=1,2, \ldots, N$. The same notation applies for 
the control input the external signals and the performance outputs $z(k+i \mid k)$.

$$
\begin{aligned}
& \overbrace{\left[\begin{array}{c}
X(k+1 \mid k) \\
X(k+2 \mid k) \\
\vdots \\
X(k+N \mid k)
\end{array}\right]}^{\hat{\mathbf{x}}}=\overbrace{\left[\begin{array}{c}
A \\
A^{2} \\
\vdots \\
A^{N}
\end{array}\right]}^{\underline{\mathcal{A}}} \overbrace{X(k)}^{\underline{\mathbf{x}}}+\overbrace{\left[\begin{array}{cccc}
B_{u} & 0 & \cdots & 0 \\
A B_{u} & B_{u} & & 0 \\
\vdots & \vdots & \ddots & \vdots \\
A^{N-1} B_{u} & A^{N-2} B_{u} & \cdots & B_{u}
\end{array}\right]}^{\underline{\underline{\underline{B}}}} \\
& +\overbrace{\left[\begin{array}{cccc}
B_{\varsigma} & 0 & \cdots & 0 \\
A B_{\varsigma} & B_{\varsigma} & & 0 \\
\vdots & \vdots & \ddots & \vdots \\
A^{N-1} B_{\varsigma} & A^{N-2} B_{\varsigma} & \cdots & B_{\varsigma}
\end{array}\right]}^{\underline{\mathcal{\varepsilon}}} \overbrace{\left[\begin{array}{c}
\varsigma(k) \\
\varsigma(k+1 \mid k) \\
\vdots \\
\varsigma(k+N-1 \mid k)
\end{array}\right]}^{\underline{\sigma}}
\end{aligned}
$$

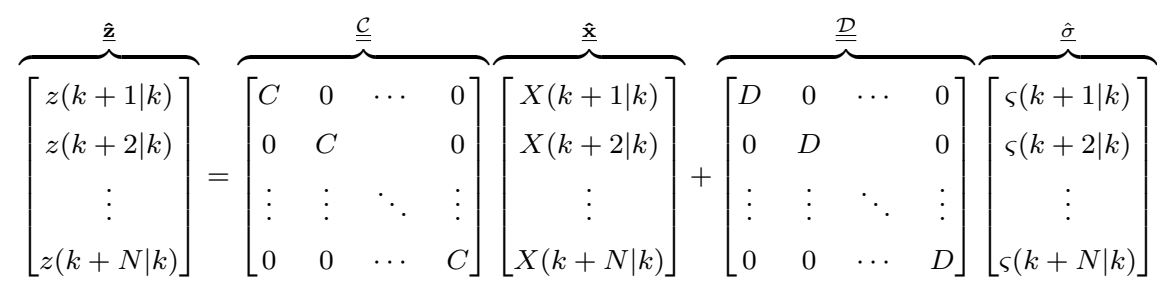

Notations in Equation $(12)$ are summarized below:

- $X(k)$ is the vector of state variables: $X(k)=[v(k), x(k)]^{T}$.

- $A$ denotes the state matrix.

- $B_{u}$ is the control input matrix containing coefficients for the desired velocity: $B_{u}=\left[\frac{\Delta t}{\tau}(1-\beta), 0,0,0\right]^{T}$.

- $u(k)$ is the controlled variable (decision variable). The only control input to the system is the desired velocity of the bus $u(k)=v_{\text {des }}(k)$.

- $B_{\varsigma}$ is the coefficient matrix for the traffic disturbance.

- $\varsigma(k)$ is a vector, collecting the uncontrolled variables of the system, i.e. the disturbance from traffic flow $v_{\text {fund }}(k)$, the two references positions: $x_{\text {des }}(k)$ and $x_{r e f}(k)$ at each time step. $\varsigma(k)=\left[v_{\text {fund }}(k), x_{\text {des }}(k), x_{\text {ref }}(k)\right]^{T}$. 
- $z(k)$ is the vector of performance outputs $z(k)=\left[z_{1}(k), z_{2}(k)\right]^{T}$.

- $C$ is the output matrix.

- $D$ is the direct feedthrough matrix of the reference trajectories.

The cost-function can be formulated with the help of Equation 13 and Equation 12 in the following form:

$$
J(k)=\frac{1}{2}\left[\underline{\hat{\mathbf{x}}}^{T} \underline{\underline{\mathcal{Q}}}_{x} \underline{\hat{\mathbf{x}}}+\underline{\hat{\mathbf{z}}}^{T} \underline{\underline{\mathcal{Q}}}_{z} \underline{\hat{\mathbf{z}}}+\underline{\mathbf{u}}^{T} \underline{\underline{\mathcal{R}}} \underline{\mathbf{u}}\right] .
$$

$\underline{\hat{\mathbf{x}}}, \underline{\mathbf{z}}$ and $\underline{\mathbf{u}}$ denote stacked vectors of the predicted states (velocity, position) performances (relative positions) and the control input (desired velocity) at each time step. The sole decision variable is the vector of desired velocities $\underline{\mathbf{u}}$ over the prediction horizon. In every time step the initial states, disturbances and parameter matrices are frozen. $\underline{\underline{\mathcal{Q}}}_{x}, \underline{\underline{\mathcal{Q}}}_{z}$ and $\underline{\underline{\mathcal{R}}}$ are diagonal, positive semidefinite weighting matrices:

$$
Q_{x}=\left[\begin{array}{cc}
q_{v} & 0 \\
0 & q_{x}
\end{array}\right], \quad Q_{z}=\left[\begin{array}{cc}
q_{x, \text { des }} & 0 \\
0 & q_{x, r e f}
\end{array}\right], \quad R=\text { const } \in \mathbb{R}^{1},
$$

where $q_{v}, q_{x}, q_{x, r e f}$ and $q_{x, \text { ref }}$ are constant weights for their respective states.

In the MPC scheme these weights are also extended for $N$ horizon: $\underline{\underline{\mathcal{Q}}}_{x}=$ $\operatorname{diag}\left(Q_{x}, Q_{x}, \ldots, Q_{x}\right) \in \mathbb{R}^{2 N \times 2 N}, \underline{\underline{\mathcal{Q}}}_{z}=\operatorname{diag}\left(Q_{z}, Q_{z}, \ldots, Q_{z}\right) \in \mathbb{R}^{2 N \times 2 N}, \underline{\underline{\mathcal{R}}}=$ $\operatorname{diag}(R, R, \ldots, R) \in \mathbb{R}^{N \times N}$.

A quadratic formula means that it penalizes both positive and negative deviations from the reference (i.e. not only late but also early arrival). $R$ adds cost to the control input. With some reformulation, the objective function to be minimized becomes:

$$
\begin{aligned}
& J(k)=\frac{1}{2} \underline{\underline{\mathbf{u}}}^{T} \overbrace{\left(\underline{\underline{\underline{\mathcal{B}}}}^{T} \underline{\underline{\mathcal{Q}}}_{x} \underline{\underline{\mathcal{B}}}+\underline{\underline{\underline{\mathcal{B}}}}^{T} \underline{\underline{\mathcal{C}}}^{T} \underline{\underline{\mathcal{Q}}} z \underline{\underline{\mathcal{C}}} \underline{\underline{\mathcal{B}}}+\underline{\underline{\mathcal{R}}}\right)}^{\Phi} \underline{\mathbf{u}}+ \\
& +\overbrace{\left(\underline{\underline{\mathbf{x}}}^{T} \underline{\underline{\mathcal{A}}}^{T} \underline{\underline{\mathcal{Q}}}_{x} \underline{\underline{\mathcal{B}}}+\underline{\sigma}^{T} \underline{\underline{\mathcal{E}}}^{T} \underline{\underline{\mathcal{Q}}}_{x} \underline{\underline{\mathcal{B}}}+\underline{\underline{x}}^{T} \underline{\underline{\mathcal{A}}}^{T} \underline{\underline{\mathcal{C}}}^{T} \underline{\underline{\mathcal{Q}}} z \underline{\underline{\mathcal{C}}} \underline{\underline{\mathcal{B}}}+\underline{\sigma}^{T} \underline{\underline{\mathcal{E}}}^{T} \underline{\underline{\mathcal{C}}}^{T} \underline{\underline{\mathcal{Q}}}_{z} \underline{\underline{\mathcal{C}}} \underline{\underline{\mathcal{B}}}+\underline{\hat{\sigma}}^{T} \underline{\underline{\mathcal{D}}}^{T} \underline{\underline{\mathcal{Q}}} z \underline{\underline{\mathcal{C}}} \underline{\underline{\mathcal{B}}}\right)}^{\Omega^{T}} .
\end{aligned}
$$

and we refer to detailed derivation in Appendix A. Finally, our control objective

$$
\min _{u}\left[\frac{1}{2} \underline{\mathbf{u}}^{T} \Phi \underline{\mathbf{u}}+\Omega^{T} \underline{\mathbf{u}}\right]
$$


subject to:

$$
\begin{gathered}
\left|z_{1}(k+N \mid k)\right|<\varepsilon, \\
\left|z_{2}(k+N \mid k)\right|<\varepsilon, \\
v_{\text {min }} \leq v_{\text {des }} \leq v_{\max } .
\end{gathered}
$$

In other words the two position errors shall be smaller than $\varepsilon$ at the last time step. $\varepsilon$ is a tunable parameter in the inequality constraints, allowing a few me-

the lower limit $v_{\min }=0 \mathrm{~km} / \mathrm{h}$, since negative velocity is not allowed, $v_{\max }$ is constrained by the legal speed limit on the link (e.g. $v_{\max }=50 \mathrm{~km} / \mathrm{h}$ ).

The above optimization problem is an input constrained quadratic programming problem which can be solved with fast and efficient solvers (Nocedal and 275 Wright, 2006). At the end of the optimization process, $N$ control input signals are obtained. This can be considered as an optimal velocity profile. However, only the first control input $u(k \mid k)$ is applied to the system. The process is then continued similarly by repeating the measurement, estimation, and optimization. Accordingly, the MPC is an online technique. Therefore, it is important to apply a sufficiently fast optimization tool and appropriate control interval.

\subsection{Weighting strategies}

In this section four different weighting strategies are proposed. The first is timetable tracking, where large weight is put on $x_{d e s}$ relative to $x_{r e f}$ in the cost function. The second strategy is headway tracking, i.e. the goal is to mimic the trajectory of the leading bus. The third one is a balanced strategy where timetable and headway references are equally important. Choosing the right control input weight is crucial. Finally, an adaptive strategy is presented incorporating varying control weights, depending on the magnitude of timetable and headway errors. If the control input is cheap ( $R$ is small) the control input resembles a bang-bang control strategy, most of the desired velocity values $v_{\text {des }}$ either $v_{\min }$ or $v_{\max }$. If the control input is expensive ( $R$ is large), the system responds slowly and the desired performance criteria (timetable and headway 
tracking) is not met. In other words, if $R$ is large, demanding high velocity would result in high cost function values. Minimum of such a function would be at small control inputs over good performance.

The controller is tuned using the inverse square law (Bryson's rule) (Bryson et al., 1979): the weights are normalized with the reciprocal of the maximum squared values of the states with $Q$ and the reciprocal of the maximum squared values of the control inputs with $R$.

$$
Q=\left[\begin{array}{cccc}
q_{1} & & & \\
& q_{2} & & \\
& & \ddots & \\
& & & q_{n}
\end{array}\right], \quad R=\frac{1}{u_{\max }^{2}}
$$

with the elements in $Q$ being:

$$
q_{i}=\frac{\gamma_{i}}{x_{\max , i}^{2}},
$$

where $q_{i}$, (the $i^{t h}$ element of the diagonal) corresponds to the $i^{t h}$ state in the state vector. Furthermore, $\gamma_{i}$ is a tunable parameter, $\sum_{i=1}^{n} \gamma_{i}=1$. In the nominator in Equation (22) $x_{\max , i}$ is the expected maximum value of the weighted state.

In the bus bunching control algorithm $Q$ is either $Q_{x}$ or $Q_{m}$ and the control weights $q_{i}$ are defined as follows: $\left\{q_{v}, q_{x}, q_{x, d e s}, q_{x, r e f}\right\}$ for their respective states in the state vector $X(k)=[v, x]^{T}$ and performance output vector $z(k)=$ $\left[z_{1}, z_{2}\right]^{T}$. The weight $R$ is related to the single control input, the desired velocity $v_{d e s}$. The corresponding weights for the four proposed control strategies are summarized in Table 1 .

In every case the selected weight for $q_{v}$ is set to zero, because it would penalize the kinetic energy of the bus (i.e. demand small velocity). Kinetic energy is weighted through $R . q_{x}$ weights the absolute position of the bus. Minimizing absolute position would be a physically unreasonable choice. Therefore the weights in $Q_{x}$ are all zero, simplifying the cost function (see the Appendix A).

Next, an adaptive weighting strategy is proposed. This control strategy uses varying control weights based on the magnitude of timetable or headway errors. 
Table 1: Control weights

\begin{tabular}{lcccc} 
& Timetable tracking & Headway tracking & Balanced & Adaptive \\
\hline$q_{x, \text { des }}$ & $3.636 \cdot 10^{-4}$ & $0.3636 \cdot 10^{-4}$ & $2.182 \cdot 10^{-4}$ & $2.182 \cdot 10^{-4}$ \\
$q_{x, \text { ref }}$ & 0 & $3.636 \cdot 10^{-4}$ & $1.818 \cdot 10^{-4}$ & $1.818 \cdot 10^{-4}$ \\
$R$ & $36 \cdot 10^{-4}$ & $36 \cdot 10^{-4}$ & $36 \cdot 10^{-4}$ & $36 \cdot 10^{-4}$ \\
\hline
\end{tabular}

By means of this adaptive weight selection it is possible to match headways more efficiently depending on the delay (timetable) and the level of bunching (headway). To this end a metric is introduced that describes the bunching level given by

$$
\zeta(k+i \mid k)=\left|\frac{z_{1}(k+i \mid k)}{z_{2}(k+i \mid k)}\right|,
$$

where $i=1, \ldots, N$ and $\zeta(k+i \mid k) \in\left[0, \zeta_{\max }\right]$. To apply this scaling other numerical considerations have to be taken into account: (i) $\zeta$ is saturated with $\zeta_{\max }=10$ to avoid enormous control weights, (ii) to circumvent division by zero $\zeta=1$ if $z_{2}=0$. The scaling parameter is calculated at the first step

315 (upon departure from a stop) and frozen for the entire prediction horizon. It is necessary to freeze the value of $\zeta$ in order to avoid algebraic loop in the solution. Thus, the optimization problem remains convex, see Appendix B. With this scaling if headway error $z_{1}$ is low $\zeta \approx 0$, timetable schedule is tracked by means of weight selection. If there is a large deviation in headway, $\zeta \gg 0$, headway error will play dominating role in the cost.

The adaptive weighting matrix becomes:

$$
Q_{z, \text { adap }}\left(z_{1}(k+i \mid k), z_{1}(k+i \mid k)\right)=\left[\begin{array}{cc}
\zeta(k+i \mid k) \cdot q_{x, \text { des }} & 0 \\
0 & q_{x, r e f}
\end{array}\right] .
$$

It is sufficient to scale only $q_{x, r e f}$, since the ratio of $q_{x, \text { ref }}$ and $q_{x, \text { des }}$ determine which objective is more important to track. In the adaptive weighting solution the same control weights are used as in the balanced strategy, see Table 1.

\subsection{Pareto Front}

The controller aims at striking a balance between headway and timetable keeping. These two objectives do not have a unique solution but a set of op- 
timal solutions. This set is called the Pareto Front (Veldhuizen and Lamont, 1998). With the Pareto analysis, we can quantify the gain of different weighting strategies.

In Section 5.3 weighting matrices $Q_{x}, Q_{z}$ and $R$ were introduced. The MPC controller was obtained by minimizing a quadratic cost function. During this optimization, two objectives were taken into account: minimizing error relative to the headway of the leading bus and minimizing headway relative to the timetable. This optimization can be recast as a multiobjective problem. The weighting matrices $Q_{x}, Q_{z}$ and $R$ are therefore decoupled as follows:

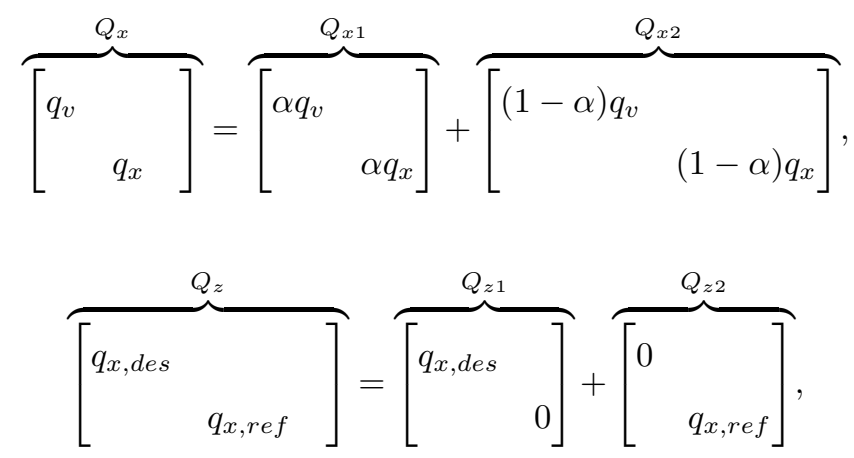

Furthermore, $R$ is split too:

$$
R=\alpha R+(1-\alpha) R=R_{1}+R_{2},
$$

where $\alpha \in[0,1]$ is a tradeoff parameter, i.e. when assuming equality between the two performances $\alpha=\frac{1}{2}$. The control weights are extended to $N$ horizon: $\underline{\underline{\mathcal{Q}_{\mathbf{x} 1}}}=\operatorname{diag}\left(Q_{x 1,1}, Q_{x 1,2}, \ldots, Q_{x 1, N}\right)$,

$\underline{\underline{\mathcal{Q}_{\mathbf{x} 2}}}=\operatorname{diag}\left(Q_{x 2,1}, Q_{x 2,2}, \ldots, Q_{x 2, N}\right)$,

$\underline{\underline{\mathcal{Q}_{\mathbf{z} 1}}}=\operatorname{diag}\left(Q_{z 1,1}, Q_{z 1,2}, \ldots, Q_{z 1, N}\right)$,

${ }_{335} \underline{\underline{\mathcal{Q}_{\mathbf{z 2} 2}}}=\operatorname{diag}\left(Q_{z 2,1}, Q_{z 2,2}, \ldots, Q_{z 2, N}\right)$, $\underline{\underline{\mathcal{R}_{1}}}=\operatorname{diag}\left(R_{1,1}, R_{1,2}, \ldots, R_{1, N}\right)$ and $\underline{\underline{\mathcal{R}_{2}}}=\operatorname{diag}\left(R_{2,1}, R_{2,2}, \ldots, R_{2, N}\right)$.

The quadratic cost function can be reformulated with the help of $\underline{\hat{x}}$ and $\underline{\mathbf{u}}$ :

$$
\begin{aligned}
J(k, N)=J_{1}(k, N)+J_{2}(k, N) & =\frac{1}{2}\left[\underline{\hat{\mathbf{x}}}^{T} \underline{\underline{\mathcal{Q}_{\mathbf{x} \mathbf{1}}}} \underline{\hat{\mathbf{x}}}+\underline{\underline{\mathbf{z}}}^{T} \underline{\underline{\mathcal{Q}_{\mathbf{z} 1}}} \underline{\hat{\mathbf{z}}}+\underline{\mathbf{u}}^{T} \underline{\underline{\mathcal{R}_{\mathbf{1}}}} \underline{\mathbf{u}}\right] \\
& +\frac{1}{2}\left[\underline{\hat{\mathbf{x}}}^{T} \underline{\underline{\mathcal{Q}_{\mathbf{x} \mathbf{2}}}} \underline{\hat{\mathbf{x}}}+\underline{\hat{\mathbf{z}}}^{T} \underline{\underline{\mathcal{Q}_{\mathbf{z} \mathbf{2}}}} \underline{\hat{\mathbf{z}}}+\underline{\underline{\mathbf{u}}}^{T} \underline{\underline{\mathcal{R}_{\mathbf{2}}}} \underline{\mathbf{u}}\right] .
\end{aligned}
$$


With the same deduction steps as in the single objective cost function (Equation (14) - (17) the cost function becomes:

$$
J(k, N)=\frac{1}{2} \underline{\mathbf{u}}^{T}\left(\Phi_{1}+\Phi_{2}\right) \underline{\mathbf{u}}+\left(\Omega_{1}^{T}+\Omega_{2}^{T}\right) \underline{\mathbf{u}} .
$$

The two sub-cost functions $J_{1}$ and $J_{2}$ will be used to demonstrate the Pareto Front. The methodology for obtaining the Pareto front is the following: from a selected initial state $x, z$ and prediction horizon $N$ an optimization is started using a genetic algorithm (Horn et al. 1994) with fixed control weights (headway, timetable or balanced). Candidate solutions of the genetic algorithm with the lowest cost will form the Pareto Front.

\subsection{Benchmark control strategies}

To show the efficiency of the model predictive strategies three additional velocity control laws are developed.

Uncontrolled: Buses do not have any control applied to them, their desired velocity is the legal speed limit. They try to leave the bus stop as soon as possible.

Holding: Buses have no velocity control but they are held at stops until their scheduled departure time.

PI control: The PI (Proportional-Integral) controller uses the two reference trajectories proposed in Section 5.1 but does not predict the desired trajectory as the MPC, only considers the actual timetable and headway tracking errors $z_{1}(k)$ and $z_{2}(k)$ respectively. The control input for the PI controller is calculated as follows:

$$
v_{\text {des }, P I}(k)=P_{\text {des }} \cdot z_{1}(k)+I_{\text {des }} \cdot \sum_{0}^{k} z_{1}(k)+P_{\text {ref }} \cdot z_{2}(k)+I_{\text {ref }} \cdot \sum_{0}^{k} z_{2}(k),
$$

with $P_{\text {des }}=0.025, I_{\text {des }}=0.001, P_{\text {ref }}=0.025, I_{\text {ref }}=0.001$ being tuning parameters for the PI controller with a balanced strategy. The controller was tuned using the Ziegler-Nichols method and also augmented with antiwindup due to the limitation of $v_{\text {des }}$ (Skogestad and Postlethwaite, 2005). 


\section{Simulation scenario}

For comparative analysis a high-fidelity traffic simulator, VISSIM, is used (VIS, 2011). The simulator can be used to generate different traffic scenarios and evaluate the developed control algorithm.

Place Figure 6 about here.

The route of Gothenburg's trunk bus line 16 between Lindholmen and Brunnsparken is modeled, see Figure6. The route is 4.3 kilometers long and includes six public transport stops. Between Lindholmen and Pumpgatan the line travels on a dedicated lane, then enters mixed traffic towards Frihamnsporten. On Göta älvbron it shares tracks with other public transport lines crossing the bridge (e.g. tram line 5 and 6). Nordstan and Brunsparken stops are also shared with other lines. Buses have priority at signalized intersections, shown in Figure 6 . The legal speed limit is $50 \mathrm{~km} / \mathrm{h}$ on the whole route. The time headway of the buses is 3 minutes. The passenger boarding and alighting volumes during peak hours at each stop is shown in Table 2 Furthermore, Table 2 summarizes the total number of on-board passengers (in passenger per hour - not for individual vehicles) is summarized after each stop. In addition, the departure times from each stop is presented starting from Lindholmen stop at 0 seconds (repeating every 3 minutes).

Table 2: Number of boarding and alighting passengers at each stop (passengers/hour), scheduled departure time (seconds)

\begin{tabular}{lllll} 
& Boarding & Alighting & On-board & Schedule \\
\hline Lindholmen & 1500 & 0 & 1500 & 0 \\
Regnbågsgatan & 600 & 75 & 2025 & 80 \\
Pumpgatan & 400 & 200 & 2225 & 160 \\
Frihamnsporten & 200 & 25 & 2400 & 330 \\
Nordstan & 400 & 600 & 2200 & 540 \\
Brunnsparken & 1500 & 1300 & 2400 & 720 \\
\hline
\end{tabular}


The proposed vehicle to transport passengers on this route is a 18.75 meters long, articulated bus. The passenger capacity is approximately 4 passengers per square-meter resulting in 135 persons passenger capacity. The ratio of standees and sitting passengers is not addressed.

During the simulations two types of disturbances are distinguished: (1) minor disturbances coming from traffic lights, other vehicles and dwell time fluctuation. (2) In order to investigate the control system under major disturbance, traffic flow is perturbed, vehicles are stopped in the middle of Göta älvbron for ten minutes (i.e. opening the bridge). The simulator is capable of generating random boarding and alighting times, serving as an additional disturbance to the system. The models described in Section 3.1 and 3.2 are used to predict the this behavior.

\section{Simulation results}

\subsection{Bus trajectories}

In the followings the proposed control algorithm is analyzed. The basis of the results is the simulation model presented in Section 6. First, one bus trajectory with balanced control strategy is explained in detail, see Figure 7 .

Place Figure 7 about here.

The blue circles represent the ideal departure times at each stop based on the bus schedule. The blue line $x_{\text {des }}$ represents the ideal trajectory for timetable tracking. The red line $x_{r e f}$ is the trajectory of the leading bus, shifted by one time headway (3 minutes). In this balanced strategy the follower (controlled) bus tries to minimize bunching (i.e. match the trajectory of the leading bus) and keep the timetable. For the next bus the reference headway will be the trajectory of the current bus. Eventually, the trajectories will converge to the original timetable while reducing bunching. The leading bus travels slowly between Lindholmen and Regnbågsgatan (due to some disturbance). The controlled bus also slows down to avoid bunching. This results in lateness but it is recovered 
by the end of the route. The bus holding strategy is also efficient in this scenario, the bus always departs from stops at the scheduled time instants but occupies stops for long periods. However, the bus holding cannot cope with severe disturbances. Figure 7 also shows the timetable and headway errors at every time instant. The velocity profile of the bus is calculated by the shrinking horizon MPC controller. The control input is ranging between $v_{\min }=0 \mathrm{~km} / \mathrm{h}$ and $v_{\max }=50 \mathrm{~km} / \mathrm{h}$. The desired velocity profile is not followed accurately because the bus has its own dynamics plus it has to adjust its own velocity to the surrounding traffic.

Figure 11 depicts the bus trajectories without velocity control where the desired speed of every bus is $50 \mathrm{~km} / \mathrm{h}$. The second bus enters the network with one minute delay (4 minutes headway). The blue circles represent the departure times at each stop based on the bus schedule. Since there is no velocity control and the buses do not wait at stops till the scheduled departure time, their arrivals and departures are out of sync. This results in bunching too, see trajectory 2-3 in Figure 11. Another example is given without velocity control but with bus holding in Figure 12 . This strategy can remedy bunching and adhere to the schedule at the cost of spending long times at the stop. For example, Brunnsparken stop is almost always occupied by a bus.

The PI control can efficiently reduce bunching and adhere to the schedule with smaller computational demand than the MPC (Figure 13). However, it cannot cope well with disturbances: for example if the leading bus was stopped by a traffic light the controlled bus will also slow down regardless what the traffic light indicates. On the other hand the MPC controllers consider a long trajectory ahead and buses can optimally adjust their velocity considering such obstacles (with the example of the traffic light - the MPC controller takes into account how long the leading bus was blocked by the traffic light).

${ }_{430}$ Next, headway tracking MPC solution is implemented (Figure 14). With this algorithm bunching is reduced, headway error is decreasing for consecutive buses. However, the buses are still out of sync with the schedule. The timetable tracking control strategy (Figure 15) can keep the schedule few head- 
ways downstream the delay. Since the timetable is periodic, headway tracking is satisfactory too. The balanced control solution (Figure 16) has the best of two worlds: the actual and reference trajectories overlap, meaning no bunching, while the timetable is kept too. The adaptive weighting method (Figure 17) can outperform the balanced control in both aspects. In the controlled simulations the first bus only obeys the timetable reference, headway reference does not exist.

\subsection{Service recovery}

In the following simulation scenario the response of each control strategy to extreme disturbance is evaluated. The traffic is stopped in the middle of Göta älvbron for ten minutes (i.e. opening the bridge). Over this period congestion

${ }_{445}$ is formed, delays increase. After, traffic is released and congestion starts to dissipate. In the sequel, we analyze the metrics of service recovery from extreme perturbation. Here, we focus on congestion dissipation speed. In Figures 1823 the space-time diagrams of the buses with different control strategies are shown. The gray lines indicate a speed, thereof the the dissipation of congestion. It starts after the bridge is opened and vehicles start to queue up before Nordstan stop. The end point of the line shows when the vehicles arrive to Nordstan with the scheduled rate (headways recover to 3 minutes). The slope of the line is the dissipation speed of the congestion and is summarized in Table 3

\begin{tabular}{ll} 
Table 3: Congestion dissipation speed $(\mathrm{m} / \mathrm{s})$ \\
Uncontrolled & 2.135 \\
Holding & 2.004 \\
PI control & 0.7268 \\
Headway tracking & 0.469 \\
Timetable tracking & 1.915 \\
Balanced & 0.608 \\
Adaptive & 0.5851 \\
\hline
\end{tabular}

In the uncontrolled and holding scenarios (Figure 18, 19) five buses are 

the bridge both bunching and timetable reliability are poor $\left(\left|z_{1}\right|\right.$ and $\left|z_{2}\right|$ are high). Since there is no way to adhere to the (static) timetable only headways are corrected gradually ( $z_{1}$ becomes small). Eventually, catching up with the timetable becomes more significant. In the multiobjective control approaches, 485

affected by the service perturbation. The buses stopped at the bridge will remain bunched. Since there is no timetable or headway objective, vehicles leave the network as fast as possible, resulting in the fastest congestion dissipation. As stated in (Newell, 1977), delays remain so high that the scheduling cannot recover from bunching. In order for the holding strategy to work in case of large disturbances, other measures, such as stop skipping, pulling out or inserting buses shall be employed. The PI controller (Figure 20 can dissipate the congestion faster than the balanced controller but the system cannot recover well from the service perturbation, the buses leave the network bunched. With headway tracking strategy (Figure 21) bunching is completely eliminated but buses arrive with large delays at the next stop, not obeying the timetable. This strategy results in slow service between the bridge and Nordstan stop in order to equalize headways. Congestion dissipation is very slow. Timetable tracking solution (Figure 22) cannot cope with the severe perturbation. The buses that got caught by the opening of the bridge stick together and cannot recover. The large difference between the desired trajectory based on the timetable and the actual trajectory (i.e. large delay) forces the velocity controller to demand maximum velocity. Therefore, in order to recover, another policy (e.g. slack times, stop skipping, dynamic timetable) has to be used. The scheduler does not make corrections due to perturbed service. The balanced technique (Figure 23) reduces bunching compared to the timetable tracking but cannot eliminate it as well as headway tracking. Recovery of the timetable takes more time compared to the timetable tracking policy. The trade-off between headway and timetable tracking can also be observed in the congestion dissipation speed. Finally, the adaptive weight approach (Figure 24) slightly outperforms the balanced control in terms of congestion dissipation. In this scenario, after releasing traffic at given enough time both timetable keeping and bunching can be remedied. 


\subsection{Headway reliability}

Table 4 compares headway reliability in the different simulation scenarios based on statistical results: Headways are compared at two sections of the network: after Frihamnsporten and after Brunnsparken stops. The mean value is similar in every case, close to the ideal headway of 180 seconds ( 3 min) except for the headway tracking and balanced control strategies after the bridge was opened. The reason is that after the traffic is released from the bridge there is a huge headway gap between two buses which corrupts the mean value. On the other hand in those strategies where headway tracking is not addressed this huge gap is counterbalanced by the small headways of the congested buses. Furthermore, headway standard deviations are smallest in the headway tracking and balanced scenarios. Finally, the Kullback-Liebler (KL) divergence is given between the ideal headway, and the simulation results, see (Kullback and Leibler. 1951). The ideal headway represents a uniform distribution with mean of 180 seconds and 0 variance. The KL distance is significantly smaller in the controlled cases compared to the uncontrolled and the holding one after the service disruption, which means headways are more uniformly distributed. The PI control works as well as the MPC solutions under in small disturbance conditions but performs significantly worse under major disturbance.

\subsection{Average passenger waiting times}

Average passenger waiting time relates directly to bus headways (Wu et al. 2017). If buses arrive irregularly, passenger waiting times will deviate more and the total waiting time will increase. Passenger arrivals at stops are generated by the simulator. In Equation (31) the average time passengers spend waiting for the bus at stop $j$ is calculated. $t_{d e p, i-1}$ and $t_{d e p, i}$ denote the departure times of bus $i-1$ and bus $i$, respectively. $N_{\text {pass }, j}(k)$ is the number of passengers present at stop $j$ at time step $k$ and $N_{\text {pass }, j}\left(t_{d e p, i}\right)$ is the terminal number of passengers boarding the $i^{t h}$ bus. $\Delta t=1$ is the sampling interval of the simulation.

$$
\bar{T}_{w, j}=\sum_{k=t_{d e p, i-1}}^{t_{d e p, i}} \frac{N_{\text {pass }, j}(k) \Delta t}{N_{\text {pass }, j}\left(t_{d e p, i}\right)} .
$$


Table 4: Statistics of the trajectories Without service perturbation

\begin{tabular}{lllllll}
\hline & \multicolumn{3}{c}{ After Frihamnsporten } & \multicolumn{3}{c}{ After Brunnsparken } \\
\hline & Mean (s) & Std (s) & KL dist. & Mean (s) & Std (s) & KL dist. \\
\hline Uncontrolled & 183.366 & 16.967 & 0.0083 & 179.629 & 67.014 & 0.0743 \\
Holding & 179.647 & 7.026 & 0.0073 & 176.290 & 27.670 & 0.0123 \\
PI control & 183.333 & 13.637 & 0.0060 & 176.000 & 34.935 & 0.0204 \\
Headway tracking & 179.130 & 17.808 & 0.0037 & 179.130 & 22.347 & 0.0057 \\
Timetable tracking & 178.706 & 7.728 & 0.0088 & 176.400 & 47.042 & 0.0180 \\
Balanced & 179.647 & 10.386 & 0.0061 & 180.403 & 31.297 & 0.0068 \\
Adaptive & 179.650 & 4.957 & 0.0036 & 180.332 & 28.451 & 0.0062 \\
\hline & & & With service perturbation & \\
\hline Uncontrolled & 184.167 & 37.307 & 0.0194 & 179.159 & 163.904 & 0.3484 \\
Holding & 181.923 & 9.962 & 0.0104 & 180.153 & 175.506 & 0.3581 \\
PI control & 182.832 & 15.077 & 0.0071 & 186.73 & 149.680 & 0.2124 \\
Headway tracking & 179.600 & 24.233 & 0.0035 & 236.450 & 134.797 & 0.1072 \\
Timetable tracking & 178.118 & 6.499 & 0.0063 & 176.401 & 141.494 & 0.2332 \\
Balanced & 179.881 & 15.530 & 0.0069 & 192.133 & 133.873 & 0.1640 \\
Adaptive & 179.400 & 5.248 & 0.0041 & 189.867 & 130.873 & 0.1588 \\
\hline
\end{tabular}

Passenger waiting time at each stop is then averaged for every bus in the in the simulation.

$$
\overline{\bar{T}}_{w, j}=\frac{1}{Y} \sum_{i=1}^{Y} \frac{\sum_{k=t_{d e p, i-1}}^{t_{\text {dep }, i}} N_{\text {pass }, j}(k) \Delta t}{N_{\text {pass }, j}\left(t_{\text {dep }, i}\right)},
$$

where $Y$ is the number of buses. Table 5 summarizes the average passenger waiting times at each stop $\overline{\bar{T}}_{w, j}$ and their standard deviation (among buses).

The average waiting time at each stop and in each control strategy is similar. Standard deviations on the other hand, are smaller in the timetable tracking, balanced and adaptive scaling scenarios. This is most apparent in the simulation scenarios with service perturbation at stops 5 (Nordstan) and 6 (Brunnsparken). This metric indirectly validates the passenger arrival and dwell time model, proposed in Section 3.1 and 3.2 too. 
Table 5: Average and standard deviation of passenger waiting times

\begin{tabular}{|c|c|c|c|c|c|c|}
\hline Stop ID & 1 & 2 & 3 & 4 & 5 & 6 \\
\hline & \multicolumn{6}{|c|}{ Without service perturbation } \\
\hline \multirow{2}{*}{ Uncontrolled } & 102.9781 & 91.5016 & 92.5255 & 91.5507 & 89.2057 & 98.8088 \\
\hline & 16.6173 & 20.3400 & 27.8874 & 16.3585 & 29.2976 & 38.4507 \\
\hline \multirow{2}{*}{ Holding } & 102.7160 & 89.6453 & 83.4922 & 83.4309 & 81.0430 & 93.9779 \\
\hline & 22.9769 & 17.3980 & 17.7942 & 15.6758 & 17.9013 & 29.1266 \\
\hline \multirow{2}{*}{ PI control } & 106.0743 & 91.1691 & 81.0872 & 86.4429 & 83.9475 & 94.0891 \\
\hline & 25.3866 & 20.0087 & 14.9044 & 23.4781 & 16.4071 & 28.7084 \\
\hline \multirow{2}{*}{$\begin{array}{l}\text { Headway } \\
\text { tracking }\end{array}$} & 106.0966 & 97.6611 & 90.6139 & 99.0809 & 92.7141 & 93.4921 \\
\hline & 23.2617 & 25.1032 & 19.5131 & 26.6081 & 26.5318 & 42.5043 \\
\hline \multirow{2}{*}{$\begin{array}{l}\text { Timetable } \\
\text { tracking }\end{array}$} & 102.6009 & 89.3517 & 87.2137 & 90.9942 & 86.8114 & 98.1383 \\
\hline & 22.3640 & 15.2167 & 18.9259 & 15.4202 & 17.4621 & 23.2008 \\
\hline \multirow{2}{*}{ Balanced } & 104.0408 & 88.7699 & 86.6075 & 90.9584 & 87.8873 & 96.8738 \\
\hline & 23.2828 & 18.1306 & 18.9966 & 17.8419 & 14.9069 & 29.1305 \\
\hline \multirow{3}{*}{ Adaptive } & 104.5393 & 89.5334 & 90.7480 & 87.8388 & 86.2397 & 96.7821 \\
\hline & 17.8173 & 15.9722 & 18.6181 & 19.8458 & 16.0954 & 17.6693 \\
\hline & \multicolumn{6}{|c|}{ With service perturbation } \\
\hline \multirow{2}{*}{ Uncontrolled } & 99.1804 & 94.6717 & 91.7990 & 91.0310 & 86.9875 & 102.4097 \\
\hline & 14.2812 & 23.1444 & 21.6361 & 24.2347 & 68.8918 & 77.8500 \\
\hline \multirow{2}{*}{ Holding } & 102.0215 & 93.6236 & 89.7799 & 86.2057 & 85.7608 & 96.5311 \\
\hline & 15.6234 & 16.1319 & 16.1889 & 17.5029 & 76.1980 & 86.4498 \\
\hline \multirow{2}{*}{ PI control } & 104.4743 & 91.2377 & 82.7137 & 92.6406 & 83.1077 & 95.8536 \\
\hline & 21.3350 & 22.3964 & 14.5604 & 23.6320 & 80.2634 & 82.9688 \\
\hline \multirow{2}{*}{$\begin{array}{l}\text { Headway } \\
\text { tracking }\end{array}$} & 106.1994 & 92.4425 & 94.0214 & 94.9517 & 87.9572 & 97.8872 \\
\hline & 24.3607 & 22.5538 & 20.4665 & 19.2216 & 62.2906 & 72.7413 \\
\hline \multirow{2}{*}{$\begin{array}{l}\text { Timetable } \\
\text { tracking }\end{array}$} & 101.4535 & 90.6727 & 83.2642 & 79.5055 & 88.7093 & 97.9377 \\
\hline & 18.4607 & 11.6870 & 14.6046 & 19.4177 & 66.1175 & 67.6487 \\
\hline \multirow{2}{*}{ Balanced } & 105.4288 & 91.5759 & 90.0413 & 90.6749 & 93.1830 & 101.9473 \\
\hline & 19.3001 & 16.6546 & 18.5899 & 22.3945 & 71.6810 & 68.9835 \\
\hline \multirow{2}{*}{ Adaptive } & 103.1233 & 89.6665 & 88.4717 & 86.4636 & 83.4796 & 95.0347 \\
\hline & 18.0314 & 18.8225 & 19.9738 & 17.6503 & 72.2092 & 65.7505 \\
\hline
\end{tabular}

\subsection{Tracking errors}

Next, average tracking errors of $x_{d e s}$ and $x_{r e f}$ are illustrated in Figure 8 for each control strategy. One bar in the plots represents one bus. The height 
of a bar is the mean absolute difference between its own trajectory and the reference $\left(x_{d e s}\right.$ or $x_{r e f}$, as in Figure 7$)$. The measure of bunching $M_{B}$ is defined as the averaged absolute headway difference for each control strategy. Similarly, measure of punctuality $M_{P}$ is the mean of the timetable errors. For each bus $i$ :

$$
\begin{aligned}
& M_{B}=\sum_{i=1}^{Y} \sum_{k_{i}=1}^{K_{i}} \frac{\left|x_{i}(k)-x_{\text {ref,i }}(k)\right|}{K_{i} \cdot Y}, \\
& M_{P}=\sum_{i=1}^{Y} \sum_{k_{i}=1}^{K_{i}} \frac{\left|x_{i}(k)-x_{\text {des,i }}(k)\right|}{K_{i} \cdot Y},
\end{aligned}
$$

where $k_{i}$ and $K_{i}$ are the entry and exit times of bus $i$ to the network respectively. $Y$ is the number of buses in the simulation.

The results are summarized in Table 6. The buses are the most punctual with timetable tracking strategy (as expected) and least punctual with headway tracking. The holding strategy fares worse than the timetable tracking in terms of punctuality because deviations from the timetable reference are only corrected at a stop. In terms of bunching, the headway tracking policy turns out to be the best.

Place Figure 8 about here.

Table 6: Measure of punctuality and bunching distance expressed as ratio compared to PI control (considered as reference $(100 \%)$ in this benchmark). Smaller number indicates better performance.

\begin{tabular}{lll} 
& Punctuality $\left(M_{P}\right)$ & Bunching $\left(M_{P}\right)$ \\
\hline Uncontrolled & $362.67(161.5 \%)$ & $165.91(119.89 \%)$ \\
Holding & $226.95(101 \%)$ & $147.13(106.3 \%)$ \\
PI control & $224.56(100 \%)$ & $138.39(100 \%)$ \\
Headway tracking & $264.82(118 \%)$ & $77.68(56.1 \%)$ \\
Timetable tracking & $181.32(80.6 \%)$ & $294.09(213 \%)$ \\
Balanced & $209.92(93.5 \%)$ & $125.24(90.5 \%)$ \\
Adaptive & $205.25(91.4 \%)$ & $78.35(56.6 \%)$ \\
\hline
\end{tabular}




\subsection{Pareto Front}

525 instant considering the three different weighting strategies (timetable tracking, headway tracking and balanced), see Figure 9. Adaptive weight approach is not shown in Figure 9. In this example the prediction horizon is $N=10$ and the states are: $x=[5.4,614.8]^{T}, z=[3.3,269.9]^{T}$. If timetable trackcontrol algorithm. The computation time needed for optimization increases as the horizon length $N$ grows, see Figure 10 . The step time of the simulation has been selected to 1 second. In the case-study, there are stops require more than 120 steps from each other and for sake of simplicity we disregard the problem of this problem if arises. We suggest to adjust the prediction time steps of the controller dynamically, based on the distance from the next stop.

Place Figure 10 about here.

\section{Conclusions and future work}

In this paper a multiobjective control strategy was presented to overcome bus bunching and improve timetable reliability. The goal with the speed advisory 
system is to choose the velocity profile of a vehicle in such a way that it keeps the timetable as well as the desired headway from the predecessor bus. To this end, a model was proposed incorporating dwell time at stops and the adverse effect of traffic. Based on the proposed linear bus following model, a receding horizon model predictive controller was formulated. The controller calculates an optimal velocity profile between the current position of the bus and the location of the next stop, taking into account its schedule and the position of the leader bus. Three different control strategies were proposed, balancing between two reference tracking objectives. The Pareto front of each control strategy was analyzed. The viability of the speed control algorithms were demonstrated with a high fidelity traffic simulator in realistic scenarios. Different metrics were proposed to compare the performance of the control strategies. Each strategy has its advantage and disadvantage: Timetable tracking solution is efficient during off-peak hours when bunching is not significant or when it is desirable to empty the network quickly after a major disturbance. Headway tracking is capable of eliminating bunching at the cost of abandoning the timetable. This strategy can work well if headways are short and buses tend to bunch - in case of short headways passengers are less likely to consult the timetable. The balanced strategy strikes equilibrium between the objective of headway and timetable tracking, granting good performance in both aspects. The adaptive scaling controller can outperform the balanced control strategy in both timetable tracking and headway tracking aspect. Deciding among the proposed control strategies brings in several factors such as passenger demand, frequency of buses, network layout, potential disturbances, etc. The controllers were compared to three benchmark strategies: uncontrolled, holding and PI controlled scenarios. The MPC controllers outperform the PI controller in the event of extreme disturbance in both timetable and headway tracking. If there are minor disturbances, the controllers are on par. In terms of passenger waiting times there is no significant difference among the control strategies.

As future research directions, we believe incorporating real-time traffic signalization explicitly in the model (i.e. considering signal stage changing times) 
can improve the accuracy of speed prediction. Moreover, combining public transport priority with bus bunching control (via traffic signals) is certainly one of the most interesting problem to consider. In addition, the effect of passenger number uncertainty over the optimal solution has to be better analyzed. Since, in the elaborated framework, uncertain passenger number can yield biased estimate of time of arrival. This further propagates to uncertain prediction horizon length, claiming for stochastic MPC. Finally, network bunching control solutions are important future works, where stability guarantees and service homogeneity on for the entire public transport network have to be ensured.

Place Figure 11 about here.

Place Figure 12 about here.

Place Figure 13 about here.

595 Place Figure 14 about here.

Place Figure 15 about here.

Place Figure 16 about here.

Place Figure 17 about here.

Place Figure 19 about here.

Place Figure 20 about here.

Place Figure 21 about here.

Place Figure 22 about here.

Place Figure 23 about here.

Place Figure 24 about here.

605

\section{Appendix A - Derivation of the cost function}

The augmented state space representation and the two performance output trajectories:

$$
\overbrace{\left[\begin{array}{l}
v(k+1) \\
x(k+1)
\end{array}\right]}^{X(k+1)}=\overbrace{\left[\begin{array}{cc}
1-\frac{\Delta t}{\tau} & 0 \\
\Delta t & 1
\end{array}\right]}^{A} \overbrace{\left[\begin{array}{c}
v(k) \\
x(k)
\end{array}\right]}^{X(k)}+\overbrace{\left[\begin{array}{c}
\frac{\Delta t}{\tau}(1-\beta) \\
0
\end{array}\right]}^{B_{u}} \overbrace{v_{\text {des }}(k)}^{u(k)}+\overbrace{\left[\begin{array}{ccc}
\beta & 0 & 0 \\
0 & 0 & 0
\end{array}\right]}^{B_{\varsigma}} \overbrace{\left[\begin{array}{c}
v_{\text {fund }}(k) \\
x_{\text {des }}(k) \\
x_{\text {ref }}(k)
\end{array}\right]}^{\varsigma(k)}
$$




$$
\overbrace{\left[\begin{array}{l}
z_{1}(k) \\
z_{2}(k)
\end{array}\right]}^{z(k)}=\overbrace{\left[\begin{array}{rr}
0 & -1 \\
0 & -1
\end{array}\right]}^{C} \overbrace{\left[\begin{array}{l}
v(k) \\
x(k)
\end{array}\right]}^{X(k)}+\overbrace{\left[\begin{array}{lll}
0 & 1 & 0 \\
0 & 0 & 1
\end{array}\right]}^{D} \overbrace{\left[\begin{array}{c}
v_{\text {fund }}(k) \\
x_{\text {des }}(k) \\
x_{\text {ref }}(k)
\end{array}\right]}^{\varsigma(k)}
$$

The state space representation in Equation 35 and the tracking performance in Equation (36) can be extended for finite $N$ horizon:

$$
\begin{aligned}
& \overbrace{\left[\begin{array}{c}
X(k+1 \mid k) \\
X(k+2 \mid k) \\
\vdots \\
X(k+N \mid k)
\end{array}\right]}^{\hat{\underline{\mathbf{x}}}}=\overbrace{\left[\begin{array}{c}
A \\
A^{2} \\
\vdots \\
A^{N}
\end{array}\right]}^{\underline{\mathcal{A}}} \overbrace{X(k)}^{\underline{\mathbf{x}}}+\overbrace{\left[\begin{array}{cccc}
B_{u} & 0 & \cdots & 0 \\
A B_{u} & B_{u} & & 0 \\
\vdots & \vdots & \ddots & \vdots \\
A^{N-1} B_{u} & A^{N-2} B_{u} & \cdots & B_{u}
\end{array}\right]}^{\underline{\underline{\mathcal{B}}}} \\
& \overbrace{\left[\begin{array}{cccc}
B_{\varsigma} & 0 & \cdots & 0 \\
A B_{\varsigma} & B_{\varsigma} & & 0 \\
\vdots & \vdots & \ddots & \vdots \\
A^{N-1} B_{\varsigma} & A^{N-2} B_{\varsigma} & \cdots & B_{\varsigma}
\end{array}\right]}^{\overbrace{\left[\begin{array}{c}
\varsigma \\
\varsigma(k) \\
\varsigma(k+1 \mid k) \\
\vdots \\
\varsigma(k+N-1 \mid k)
\end{array}\right]}^{\sigma}}
\end{aligned}
$$

$$
\overbrace{\left[\begin{array}{c}
z(k+1 \mid k) \\
z(k+2 \mid k) \\
\vdots \\
z(k+N \mid k)
\end{array}\right]}^{\underline{\hat{\mathbf{z}}}}=\overbrace{\left[\begin{array}{cccc}
C & 0 & \cdots & 0 \\
0 & C & & 0 \\
\vdots & \vdots & \ddots & \vdots \\
0 & 0 & \cdots & C
\end{array}\right]}^{\underline{\underline{\mathcal{C}}}} \overbrace{\left[\begin{array}{c}
X(k+1 \mid k) \\
X(k+2 \mid k) \\
\vdots \\
X(k+N \mid k)
\end{array}\right]}^{\hat{\underline{\hat{x}}}}+\overbrace{\left[\begin{array}{cccc}
D & 0 & \cdots & 0 \\
0 & D & & 0 \\
\vdots & \vdots & \ddots & \vdots \\
0 & 0 & \cdots & D
\end{array}\right]\left[\begin{array}{c}
\varsigma(k+1 \mid k) \\
\varsigma(k+2 \mid k) \\
\vdots \\
\varsigma(k+N \mid k)
\end{array}\right]}^{\underline{\underline{\mathcal{D}}}}
$$

Note that in Equation (38) the external signals are shifted by one prediction step $(\underline{\sigma}$ and $\underline{\hat{\sigma}})$. Notations in $(38)$ are described in Section 5.2 .

The cost-function can be formulated with the help of Equation 38 and Equation (37) in the following form:

$$
J(k)=\frac{1}{2}\left[\underline{\hat{\mathbf{x}}}^{T} \underline{\underline{\mathcal{Q}}}_{x} \underline{\hat{\mathbf{x}}}+\underline{\hat{\mathbf{z}}}^{T} \underline{\underline{\mathcal{Q}}}_{z} \underline{\hat{\mathbf{z}}}+\underline{\mathbf{u}}^{T} \underline{\underline{\mathcal{R}}} \underline{\mathbf{u}}\right] .
$$

$\underline{\hat{\mathbf{x}}}, \underline{\hat{\mathbf{z}}}$ and $\underline{\mathbf{u}}$ denote stacked vectors of the predicted states (velocity, position) performances (relative positions) and the control input (desired velocity) at 
each time step. $\underline{\underline{\mathcal{Q}}}_{x}, \underline{\underline{\mathcal{Q}}}_{z}$ and $\underline{\underline{\mathcal{R}}}$ are diagonal, positive semi-definite weighting matrices:

$$
Q_{x}=\left[\begin{array}{cc}
q_{v} & 0 \\
0 & q_{x}
\end{array}\right], \quad Q_{z}=\left[\begin{array}{cc}
q_{x, \text { des }} & 0 \\
0 & q_{x, \text { ref }}
\end{array}\right], \quad R=\text { const } \in \mathbb{R}^{1},
$$

where $q_{v}, q_{x}, q_{x, r e f}$ and $q_{x, \text { ref }}$ are constant weights for their respective states.

In the MPC scheme these weights are also extended for $N$ horizon: $\underline{\mathcal{Q}}_{x}=$ $\operatorname{diag}\left(Q_{x}, Q_{x}, \ldots, Q_{x}\right) \in \mathbb{R}^{2 N \times 2 N}, \underline{\underline{\mathcal{Q}}}_{z}=\operatorname{diag}\left(Q_{z}, Q_{z}, \ldots, Q_{z}\right) \in \mathbb{R}^{2 N \times 2 N}, \underline{\underline{\mathcal{R}}}=$ $\operatorname{diag}(R, R, \ldots, R) \in \mathbb{R}^{N \times N}$.

First, insert (37) and (38) into (39):

$$
\begin{aligned}
J(k) & =\frac{1}{2}\left[(\underline{\underline{\mathcal{A}}} \underline{\mathbf{x}}+\underline{\underline{\mathcal{B}}} \underline{\mathbf{u}}+\underline{\underline{\mathcal{E}}} \underline{\underline{\sigma}})^{T} \underline{\underline{\mathcal{O}}}_{x}(\underline{\underline{\mathcal{A}}} \underline{\mathbf{x}}+\underline{\underline{\mathcal{B}}} \underline{\mathbf{u}}+\underline{\underline{\mathcal{E}}} \underline{\sigma})\right. \\
& +(\underline{\underline{\mathcal{C}}}\{\underline{\underline{\mathcal{A}}} \underline{\mathbf{x}}+\underline{\underline{\mathcal{B}}} \underline{\mathbf{u}}+\underline{\underline{\mathcal{E}}} \underline{\sigma}\}+\underline{\underline{\mathcal{D}}} \underline{\underline{\hat{\sigma}}})^{T} \underline{\underline{\mathcal{O}}}_{z}(\underline{\underline{\mathcal{C}}}\{\underline{\underline{\mathcal{A}}} \underline{\underline{\mathbf{x}}}+\underline{\underline{\mathcal{B}}} \underline{\mathbf{u}}+\underline{\underline{\mathcal{E}}} \underline{\sigma}\}+\underline{\underline{\mathcal{D}}} \underline{\hat{\sigma}}) \\
& \left.+\underline{\mathbf{u}^{T}} R \underline{\mathbf{u}}\right] .
\end{aligned}
$$

Then perform the multiplications $\underline{\underline{\mathbf{x}}}^{T} \underline{\underline{\mathcal{Q}}}_{x} \underline{\hat{\mathbf{x}}}$ and $\underline{\underline{\mathbf{z}}}^{T} \underline{\underline{\mathcal{Q}}}_{z} \hat{\mathbf{z}}$ :

$$
\begin{aligned}
& J(k)=\frac{1}{2}\left[\underline{\mathbf{x}}^{T} \underline{\underline{\mathcal{A}}}^{T} \underline{\underline{\mathcal{O}}}_{x} \underline{\underline{\mathcal{A}}} \underline{\mathbf{x}}+\underline{\mathbf{x}}^{T} \underline{\underline{\mathcal{A}}}^{T} \underline{\underline{\mathcal{Q}}}_{x} \underline{\underline{\mathcal{B}}} \underline{\underline{\mathbf{u}}}+\underline{\mathbf{x}}^{T} \underline{\underline{\mathcal{A}}}^{T} \underline{\underline{\mathcal{Q}}}_{x} \underline{\underline{\mathcal{E}}} \underline{\underline{\sigma}}\right. \\
& +\underline{\mathbf{u}}^{T} \underline{\underline{\mathcal{B}}}^{T} \underline{\underline{\mathcal{Q}}}_{x} \underline{\underline{\mathcal{A}}} \underline{\mathbf{x}}+\underline{\mathbf{u}}^{T} \underline{\underline{\mathcal{B}}}^{T} \underline{\underline{\mathcal{Q}}}_{x} \underline{\underline{\mathcal{B}}} \underline{\mathbf{u}}+\underline{\mathbf{u}}^{T} \underline{\underline{\mathcal{B}}}^{T} \underline{\underline{\mathcal{Q}}}_{x} \underline{\underline{\mathcal{E}}} \underline{\underline{\sigma}} \\
& +\underline{\sigma}^{T} \underline{\underline{\mathcal{E}}}^{T} \underline{\underline{\mathcal{Q}}}_{x} \underline{\underline{\mathcal{A}}} \underline{\mathbf{x}}+\underline{\sigma}^{T} \underline{\underline{\mathcal{E}}}^{T} \underline{\underline{\mathcal{Q}}}_{x} \underline{\underline{\mathcal{B}}} \underline{\mathbf{u}}+\underline{\sigma}^{T} \underline{\underline{\mathcal{E}}}^{T} \underline{\underline{\mathcal{Q}}}_{x} \underline{\underline{\mathcal{E}}} \underline{\sigma} \\
& +\underline{\mathbf{x}}^{T} \underline{\underline{\mathcal{A}}}^{T} \underline{\underline{\mathcal{C}}}^{T} \underline{\underline{\mathcal{Q}}} z \underline{\underline{\mathcal{C}}} \underline{\underline{\mathcal{A}}} \underline{\mathbf{x}}+\underline{\mathbf{x}}^{T} \underline{\underline{\mathcal{A}}}^{T} \underline{\underline{\mathcal{C}}}^{T} \underline{\underline{\mathcal{Q}}} z \underline{\underline{\mathcal{C}}} \underline{\underline{\mathcal{B}}} \underline{\mathbf{u}}+\underline{\mathbf{x}}^{T} \underline{\underline{\mathcal{A}}}^{T} \underline{\underline{\mathcal{C}}}^{T} \underline{\underline{\mathcal{Q}}} z \underline{\underline{\mathcal{C}}} \underline{\underline{\mathcal{E}}} \underline{\sigma}+\underline{\mathbf{x}}^{T} \underline{\underline{\mathcal{A}}}^{T} \underline{\underline{\mathcal{C}}}^{T} \underline{\underline{\mathcal{Q}}} z \underline{\underline{\mathcal{D}}} \underline{\underline{\sigma}} \\
& +\underline{\mathbf{u}}^{T} \underline{\underline{\mathcal{B}}}^{T} \underline{\underline{\mathcal{C}}}^{T} \underline{\underline{\mathcal{Q}}}_{z} \underline{\underline{\mathcal{C}}} \underline{\underline{\mathcal{A}}} \underline{\mathbf{x}}+\underline{\mathbf{u}}^{T} \underline{\underline{\mathcal{B}}}^{T} \underline{\underline{\mathcal{C}}}^{T} \underline{\underline{\mathcal{Q}}}_{z} \underline{\underline{\mathcal{C}}} \underline{\underline{\mathcal{B}}} \underline{\mathbf{u}}+\underline{\mathbf{u}}^{T} \underline{\underline{\mathcal{B}}}^{T} \underline{\underline{\mathcal{C}}}^{T} \underline{\underline{\mathcal{Q}}} z \underline{\underline{\mathcal{C}}} \underline{\underline{\mathcal{E}}} \underline{\underline{\sigma}}+\underline{\mathbf{u}}^{T} \underline{\underline{\mathcal{B}}}^{T} \underline{\underline{\mathcal{C}}}^{T} \underline{\underline{\mathcal{Q}}}_{z} \underline{\underline{\mathcal{D}}} \hat{\underline{\sigma}} \\
& +\underline{\sigma}^{T} \underline{\underline{\mathcal{E}}}^{T} \underline{\underline{\mathcal{C}}}^{T} \underline{\underline{\mathcal{Q}}} z \underline{\underline{\mathcal{C}}} \underline{\underline{\mathcal{A}}} \underline{\mathbf{x}}+\underline{\sigma}^{T} \underline{\underline{\mathcal{E}}}^{T} \underline{\underline{\mathcal{C}}}^{T} \underline{\underline{\mathcal{Q}}} z \underline{\underline{\mathcal{C}}} \underline{\underline{\mathcal{B}}} \underline{\mathbf{u}}+\underline{\sigma}^{T} \underline{\underline{\mathcal{E}}}^{T} \underline{\underline{\mathcal{C}}}^{T} \underline{\underline{\mathcal{Q}}} z \underline{\underline{\mathcal{C}}} \underline{\underline{\mathcal{E}}} \underline{\sigma}+\underline{\sigma}^{T} \underline{\underline{\mathcal{E}}}^{T} \underline{\underline{\mathcal{C}}}^{T} \underline{\underline{\mathcal{O}}}_{z} \underline{\underline{\mathcal{D}}} \underline{\underline{\sigma}} \\
& +\underline{\underline{\hat{\sigma}}}^{T} \underline{\underline{\mathcal{D}}}^{T} \underline{\underline{\mathcal{Q}}} z \underline{\underline{\mathcal{C}}} \underline{\underline{\mathcal{A}}} \underline{\mathbf{x}}+\underline{\hat{\sigma}}^{T} \underline{\underline{\mathcal{D}}}^{T} \underline{\underline{\mathcal{Q}}} z \underline{\underline{\mathcal{C}}} \underline{\underline{\mathcal{B}}} \underline{\mathbf{u}}+\underline{\hat{\hat{\sigma}}}^{T} \underline{\underline{\mathcal{D}}}^{T} \underline{\underline{\mathcal{Q}}} z \underline{\underline{\mathcal{C}}} \underline{\underline{\mathcal{E}}} \underline{\underline{\sigma}}+\underline{\hat{\sigma}}^{T} \underline{\underline{\mathcal{D}}}^{T} \underline{\underline{\mathcal{Q}}} z \underline{\underline{\mathcal{D}}} \\
& \left.+\underline{\mathbf{u}}^{T} R \underline{\mathbf{u}}\right] \text {. }
\end{aligned}
$$

Next, organize the terms as coefficients of $\underline{\mathbf{u}}, \underline{\mathbf{u}}^{T}$ and $\underline{\mathbf{u}}^{T}(\star) \underline{\mathbf{u}}$ :

$$
\begin{aligned}
& J(k)=\frac{1}{2}\left[\left(\underline{\underline{x}}^{T} \underline{\underline{\mathcal{A}}}^{T} \underline{\underline{\mathcal{Q}}}_{x} \underline{\underline{\mathcal{B}}}+\underline{\sigma}^{T} \underline{\underline{\mathcal{E}}}^{T} \underline{\underline{\mathcal{Q}}}_{x} \underline{\underline{\mathcal{B}}}+\underline{\mathbf{x}}^{T} \underline{\underline{\mathcal{A}}}^{T} \underline{\underline{\mathcal{C}}}^{T} \underline{\underline{\mathcal{Q}}}_{z} \underline{\underline{\mathcal{C}}} \underline{\underline{\mathcal{B}}}+\underline{\sigma}^{T} \underline{\underline{\mathcal{E}}}^{T} \underline{\underline{\mathcal{C}}}^{T} \underline{\underline{\mathcal{Q}}}_{z} \underline{\underline{\mathcal{C}}} \underline{\underline{\mathcal{B}}}+\underline{\hat{\sigma}}^{T} \underline{\underline{\mathcal{D}}}^{T} \underline{\underline{\mathcal{Q}}} z \underline{\underline{\mathcal{C}}} \underline{\underline{\mathcal{B}}}\right) \underline{\mathbf{u}}\right. \\
& +\underline{\mathbf{u}}^{T}\left(\underline{\underline{\mathcal{B}}}^{T} \underline{\underline{\mathcal{Q}}}_{x} \underline{\underline{\mathcal{A}}} \underline{\mathbf{x}}+\underline{\underline{\mathcal{B}}}^{T} \underline{\underline{\mathcal{Q}}}_{x} \underline{\underline{\mathcal{E}}} \underline{\underline{\sigma}}+\underline{\underline{\mathcal{B}}}^{T} \underline{\underline{\mathcal{C}}}^{T} \underline{\underline{\mathcal{Q}}}_{z} \underline{\underline{\mathcal{C}}} \underline{\underline{\mathcal{A}}} \underline{\mathbf{x}}+\underline{\underline{\mathcal{B}}}^{T} \underline{\underline{\mathcal{C}}}^{T} \underline{\underline{\mathcal{Q}}}_{z} \underline{\underline{\mathcal{C}}} \underline{\underline{\mathcal{E}}} \underline{\sigma}+\underline{\underline{\mathcal{B}}}^{T} \underline{\underline{\mathcal{C}}}^{T} \underline{\underline{\mathcal{Q}}} z \underline{\underline{\mathcal{D}}} \underline{\underline{\hat{\sigma}}}\right) \\
& +\underline{\mathbf{u}}^{T}\left(\underline{\underline{\mathcal{B}}}^{T} \underline{\underline{\mathcal{O}}}_{x} \underline{\underline{\mathcal{B}}}+\underline{\underline{\mathcal{B}}}^{T} \underline{\underline{\mathcal{C}}}^{T} \underline{\underline{\mathcal{O}}}_{z} \underline{\underline{\mathcal{C}}} \underline{\underline{\mathcal{B}}}+R\right) \underline{\mathbf{u}} \\
& +\left(\underline{\mathbf{x}}^{T} \underline{\underline{\mathcal{A}}}^{T} \underline{\underline{\mathcal{Q}}}_{x} \underline{\underline{\mathcal{A}}} \underline{\mathbf{x}}+\underline{\mathbf{x}}^{T} \underline{\underline{\mathcal{A}}}^{T} \underline{\underline{\mathcal{Q}}}_{x} \underline{\underline{\mathcal{E}}} \underline{\sigma}+\underline{\sigma}^{T} \underline{\underline{\mathcal{E}}}^{T} \underline{\underline{\mathcal{Q}}}_{x} \underline{\underline{\mathcal{A}}} \underline{\mathbf{x}}+\underline{\sigma}^{T} \underline{\underline{\mathcal{E}}}^{T} \underline{\underline{\mathcal{Q}}}_{x} \underline{\underline{\mathcal{E}}} \underline{\underline{\mathcal{O}}}\right. \\
& +\underline{\mathbf{x}}^{T} \underline{\underline{\mathcal{A}}}^{T} \underline{\underline{\mathcal{C}}}^{T} \underline{\underline{\mathcal{Q}}} z \underline{\underline{\mathcal{C}}} \underline{\underline{\mathcal{A}}} \underline{\mathbf{x}}+\underline{\mathbf{x}}^{T} \underline{\underline{\mathcal{A}}}^{T} \underline{\underline{\mathcal{C}}}^{T} \underline{\underline{\mathcal{Q}}} z \underline{\underline{\mathcal{C}}} \underline{\underline{\mathcal{E}}} \underline{\sigma}+\underline{\mathbf{x}}^{T} \underline{\underline{\mathcal{A}}}^{T} \underline{\underline{\mathcal{C}}}^{T} \underline{\underline{\mathcal{Q}}} z \underline{\underline{\mathcal{D}}} \underline{\underline{\hat{\sigma}}}+\underline{\sigma}^{T} \underline{\underline{\mathcal{E}}}^{T} \underline{\underline{\mathcal{C}}}^{T} \underline{\underline{\mathcal{Q}}} z \underline{\underline{\mathcal{C}}} \underline{\underline{\mathcal{A}}} \underline{\mathbf{x}} \\
& \left.\left.+\underline{\sigma}^{T} \underline{\underline{\mathcal{E}}}^{T} \underline{\underline{\mathcal{C}}}^{T} \underline{\underline{\mathcal{Q}}} z \underline{\underline{\mathcal{C}}} \underline{\underline{\mathcal{E}}} \underline{\underline{\sigma}}+\underline{\underline{\sigma}}^{T} \underline{\underline{\mathcal{E}}}^{T} \underline{\underline{\mathcal{C}}}^{T} \underline{\underline{\mathcal{Q}}} z \underline{\underline{\mathcal{D}}} \underline{\underline{\hat{\sigma}}}+\underline{\hat{\sigma}}^{T} \underline{\underline{\mathcal{D}}}^{T} \underline{\underline{\mathcal{Q}}}_{z} \underline{\underline{\mathcal{C}}} \underline{\underline{\mathcal{A}}} \underline{\underline{\mathbf{x}}}+\underline{\hat{\sigma}}^{T} \underline{\underline{\mathcal{D}}}^{T} \underline{\underline{\mathcal{Q}}} z \underline{\underline{\mathcal{C}}} \underline{\underline{\mathcal{E}}} \underline{\underline{\sigma}}+\underline{\hat{\sigma}}^{T} \underline{\underline{\mathcal{D}}}^{T} \underline{\underline{\mathcal{Q}}} z \underline{\underline{\mathcal{D}}} \underline{\underline{\sigma}}\right)\right] .
\end{aligned}
$$


The first and second terms of Equation (43) are equal. Furthermore, the last term is constant and can be removed from $J(k)$. The objective function shall be minimized and a constant term does not influence the outcome of the minimization problem, just offsets the cost. The objective function to be minimized becomes:

$$
\begin{array}{r}
J(k)=\frac{1}{2} \underline{\underline{\mathbf{u}}}^{T}\left(\underline{\underline{\mathcal{B}}}^{T} \underline{\underline{\mathcal{Q}}}_{x} \underline{\underline{\mathcal{B}}}+\underline{\underline{\mathcal{B}}}^{T} \underline{\underline{\mathcal{C}}}^{T} \underline{\underline{\mathcal{Q}}} z \underline{\underline{\mathcal{C}}} \underline{\underline{\mathcal{B}}}+\underline{\underline{\mathcal{R}}}\right) \underline{\mathbf{u}} \\
+\left(\underline{\mathbf{x}}^{T} \underline{\underline{\mathcal{A}}}^{T} \underline{\underline{\mathcal{Q}}}_{x} \underline{\underline{\mathcal{B}}}+\underline{\sigma}^{T} \underline{\underline{\mathcal{E}}}^{T} \underline{\underline{\mathcal{Q}}}_{x} \underline{\underline{\mathcal{B}}}+\underline{\mathbf{x}}^{T} \underline{\underline{\mathcal{A}}}^{T} \underline{\underline{\mathcal{C}}}^{T} \underline{\underline{\mathcal{Q}}} z \underline{\underline{\mathcal{C}}} \underline{\underline{\mathcal{B}}}+\underline{\sigma}^{T} \underline{\underline{\mathcal{E}}}^{T} \underline{\underline{\mathcal{C}}}^{T} \underline{\underline{\mathcal{Q}}} z \underline{\underline{\mathcal{C}}} \underline{\underline{\mathcal{B}}}+\underline{\hat{\sigma}}^{T} \underline{\underline{\mathcal{D}}}^{T} \underline{\underline{\mathcal{Q}}} z \underline{\underline{\mathcal{C}}} \underline{\underline{\mathcal{B}}}\right) \underline{\mathbf{u}} .
\end{array}
$$

In addition, weights in $\underline{\underline{\mathcal{Q}}}_{x}$ are all selected to be zero, refer to Section 5.3. Therefore, the cost function can be simplified:

$$
\begin{array}{r}
J(k)=\frac{1}{2} \underline{\mathbf{u}}^{T} \overbrace{\left(\underline{\underline{\mathcal{B}}}^{T} \underline{\underline{\mathcal{C}}}^{T} \underline{\underline{\mathcal{Q}}} z \underline{\underline{\mathcal{C}}} \underline{\underline{\mathcal{B}}}+\underline{\underline{\mathcal{R}}}\right)}^{\Phi} \underline{\mathbf{u}} \\
+\overbrace{\left(\underline{\underline{\mathbf{x}}}^{T} \underline{\underline{\mathcal{A}}}^{T} \underline{\underline{\mathcal{C}}}^{T} \underline{\underline{\mathcal{Q}}} z \underline{\underline{\mathcal{C}}} \underline{\underline{\mathcal{B}}}+\underline{\sigma}^{T} \underline{\underline{\mathcal{E}}}^{T} \underline{\underline{\mathcal{C}}}^{T} \underline{\underline{\mathcal{Q}}} z \underline{\underline{\mathcal{C}}} \underline{\underline{\mathcal{B}}}+\underline{\underline{\hat{\sigma}}}^{T} \underline{\underline{\mathcal{D}}}^{T} \underline{\underline{\mathcal{Q}}} z \underline{\underline{\mathcal{C}}} \underline{\underline{\mathcal{B}}}\right)}^{\underline{\mathbf{u}}} .
\end{array}
$$

Finally,

$$
J(k)=\frac{1}{2} \underline{\mathbf{u}}^{T} \Phi \underline{\mathbf{u}}+\Omega^{T} \underline{\mathbf{u}} .
$$

\section{Appendix B - Proving the convexity of the control problem}

Convexity of the problem is guaranteed by the positive definiteness of the quadratic term $\Phi$ (i.e. $\Phi \succ 0)$ (Horn and Johnson, 1990).

Theorem: Positive semidefiniteness and positive definiteness are defined as

follows: any symmetric $n \times n$ real matrix $\underline{\underline{\Lambda}}$ is said to be positive semidefinite if $\underline{u}^{T} \underline{\underline{\boldsymbol{\Lambda}}} \underline{u} \geq 0$, and positive definite if $\underline{u}^{T} \underline{\underline{\boldsymbol{\Lambda}}} \underline{u}>0$ for any non-zero vector $\underline{u}$ of $n$ real numbers.

\section{Proof:}

In the adaptive control strategy

$$
Q_{z, \text { adap }}\left(z_{1}(k+i \mid k), z_{1}(k+i \mid k)\right)=\left[\begin{array}{cc}
\zeta(k+i \mid k) \cdot q_{x, \text { des }} & 0 \\
0 & q_{x, \text { ref }}
\end{array}\right]
$$


is a positive definite matrix: $q_{x, \text { des }}$ and $q_{x, r e f}$ are positive numbers (see Table 1) and

$$
\zeta(k+i \mid k)=\left|\frac{z_{1}(k+i \mid k)}{z_{2}(k+i \mid k)}\right|
$$

is greater than zero due to the absolute value and bounded by an upper limit

$\zeta_{\max }$. Positive definiteness is guaranteed in every point. In addition, the rate of change of the $Q_{z, \text { adap }}$ cannot be infinitely large.

Next, the positive semidefiniteness of

$$
\Phi=\underline{\underline{\mathcal{B}}}^{T} \underline{\underline{\mathcal{C}}}^{T} \underline{\underline{\mathcal{Q}}}_{z, \text { adap }} \underline{\underline{\mathcal{C}}} \underline{\underline{\mathcal{B}}}+\underline{\underline{\mathcal{R}}}
$$

is shown.

If a matrix $\underline{\underline{\boldsymbol{\Gamma}}}$ has only real entries, then the product $\underline{\underline{\boldsymbol{\Gamma}}}^{T} \underline{\underline{\boldsymbol{\Gamma}}}$ gives a positive semidefinite matrix. As $\underline{\underline{\mathcal{Q}}}_{z, \text { adap }}$ was proven to be a positive definite diagonal matrix:

$$
\left(\underline{\underline{\mathcal{B}}} \underline{\underline{\mathcal{C}}} \underline{\underline{\mathcal{Q}}}_{z, \text { adap }}^{\frac{1}{2}}\right)^{T}\left(\underline{\underline{\mathcal{Q}}}_{z, \text { adap }}^{\frac{1}{2}} \underline{\underline{\mathcal{B}}}\right) \succeq 0
$$

\section{Acknowledgements}

This paper was supported by the ÚNKP-17-3-I New National Excellence Program of the Ministry of Human Capacities of Hungary and by the János Bolyai Research Scholarship of the Hungarian Academy of Sciences. B. Kulcsár acknowledges the support of Transport Area of Advance, a new initiative at Chalmers University of Technology, Gothenburg, Sweden. The authors thank to the 3 anonymous Reviewers for their very detailed and constructive criticism.

References

, 2011. VISSIM 5.30-05 User Manual. PTV. Stumpfstrasse 1, D-76131 Karlsruhe, Germany.

Ampountolas, K., Kring, M., 2015. Mitigating bunching with bus-following models and bus-to-bus cooperation, in: IEEE 18th International Conference on Intelligent Transportation Systems (ITSC), 15 - 18 September, 2015, Las Palmas, Spain. pp. 60-65. 
Andres, M., Nair, R., 2017. A predictive-control framework to address bus bunching. Transportation Research Part B: Methodological 104, 123-148.

Ap. Sorratini, J., Liu, R., Sinha, S., 2008. Assessing bus transport reliability using micro-simulation. Transportation Planning and Technology 31, 303324.

Bando, M., Hasebe, K., Nakayama, A., Shibata, A., Sugiyamai, Y., 1995. Dynamical model of traffic congestion and numerical simulation. Physical review E 51, 1035-1043.

Bartholdi, J.J., Eisenstein, D.D., 2012. A self-coördinating bus route to resist bus bunching. Transportation Research Part B: Methodological 46, 481-491.

Van den Berg, M., Hegyi, A., De Schutter, B., Hellendoorn, J., 2003. A macroscopic traffic flow model for integrated control of freeway and urban traffic networks, in: Decision and Control, 2003. Proceedings. 42nd IEEE Conference on, IEEE. pp. 2774-2779.

Bryson, A.E., Ho, Y.C., Siouris, G.M., 1979. Applied optimal control: Optimization, estimation, and control. IEEE Transactions on Systems, Man, and Cybernetics 9, 366-367.

Cats, O., Larijani, A., Ólafsdóttir, Á., Burghout, W., Andreasson, I., Koutsopoulos, H., 2012. Bus-holding control strategies: simulation-based evaluation and guidelines for implementation. Transportation Research Record: Journal of the Transportation Research Board , 100-108.

Daganzo, C.F., 2009. A headway-based approach to eliminate bus bunching: Systematic analysis and comparisons. Transportation Research Part B: Methodological 43, 913-921.

Daganzo, C.F., Geroliminis, N., 2008. An analytical approximation for the macroscopic fundamental diagram of urban traffic. Transportation Research Part B: Methodological 42, 771-781. 
Daganzo, C.F., Pilachowski, J., 2011. Reducing bunching with bus-to-bus cooperation. Transportation Research Part B: Methodological 45, 267-277.

Dessouky, M., Hall, R., Zhang, L., Singh, A., 2003. Real-time control of buses for schedule coordination at a terminal. Transportation Research Part A: Policy and Practice 37, 145-164.

Estrada, M., Mensión, J., Aymamí, J.M., Torres, L., 2016. Bus control strategies in corridors with signalized intersections. Transportation Research Part C: Emerging Technologies 71, 500-520.

Fonzone, A., Schmöcker, J.D., Liu, R., 2015. A model of bus bunching under reliability-based passenger arrival patterns. Transportation Research Procedia 7, 276-299.

Helbing, D., Tilch, B., 1998. Generalized force model of traffic dynamics. Physical review E 58, 133.

Holroyd, E.M., Scraggs, D.A., 1966. Waiting times for buses in central London. volume 8. Printerhall.

Hoogendoorn, S.P., Bovy, P.H., 2001. State-of-the-art of vehicular traffic flow modelling. Proceedings of the Institution of Mechanical Engineers, Part I: Journal of Systems and Control Engineering 215, 283-303.

Horn, J., Nafpliotis, N., Goldberg, D.E., 1994. A niched pareto genetic algorithm for multiobjective optimization, in: Evolutionary Computation, 1994. IEEE World Congress on Computational Intelligence., Proceedings of the First IEEE Conference on, IEEE. pp. 82-87.

Horn, R.A., Johnson, C.R., 1990. Matrix analysis. Cambridge university press.

Jiang, F., Cacchiani, V., Toth, P., 2017. Train timetabling by skip-stop planning in highly congested lines. Transportation Research Part B: Methodological 104, 149-174. 
lliffe, J.K., Hutchinson, T.P., 1975. A behavioural explanation of the association between bus and passenger arrivals at a bus stop. Transportation Science 9, 248-282.

Kesting, A., Treiber, M., Schönhof, M., Helbing, D., 2008. Adaptive cruise control design for active congestion avoidance. Transportation Research Part C: Emerging Technologies 16, 668-683.

Kittelson, Associates, Administration, U.S.F.T., Program, T.C.R., Corporation, T.D., 2003. Transit capacity and quality of service manual. volume 100. Transportation Research Board.

Kullback, S., Leibler, R.A., 1951. On information and sufficiency. The annals of mathematical statistics $22,79-86$.

Maciejowski, J.M., 2002. Predictive control: with constraints. Prentice Hall, Harlow, UK.

Mandelzys, M., Hellinga, B., 2010. Identifying causes of performance issues in bus schedule adherence with automatic vehicle location and passenger count data. Transportation Research Record: Journal of the Transportation Research Board , 9-15.

Nesheli, M.M., Ceder, A.A., Liu, T., 2015. A robust, tactic-based, real-time framework for public-transport transfer synchronization. Transportation Research Procedia 9, 246-268.

705

Newell, G., 1977. Unstable brownian motion of a bus trip, in: Statistical Mechanics and Statistical Methods in Theory and Application. Springer, pp. $645-667$.

Newell, G.F., Potts, R.B., 1964. Maintaining a bus schedule, in: Australian Road Research Board (ARRB) 2nd Conference.

710 Nocedal, J., Wright, S.J., 2006. Numerical optimization. 2 ed., Springer. 
O’Flaherty, C.A., Mangan, D.O., 1970. Bus passenger waiting times in greater manchester. Traffic Engineering and Control 11, 419-421.

Pilachowski, J.M., 2009. An approach to reducing bus bunching. University of California, Berkeley.

715 Rahman, M.M., Wirasinghe, S., Kattan, L., 2018. Analysis of bus travel time distributions for varying horizons and real-time applications. Transportation Research Part C: Emerging Technologies 86, 453-466.

Rajbhandari, R., Chien, S., Daniel, J., 2003. Estimation of bus dwell times with automatic passenger counter information. Transportation Research Record: Journal of the Transportation Research Board , 120-127.

Skogestad, S., Postlethwaite, I., 2005. Multivariable Feedback Control: Analysis and Design. Wiley.

Veldhuizen, D.A.V., Lamont, G.B., 1998. Evolutionary computation and convergence to a pareto front, in: Genetic Programming 1998 Conference, 22-25 725 July, 1998, Madison, WI, USA. pp. 221-228.

Wu, W., Liu, R., Jin, W., 2017. Modelling bus bunching and holding control with vehicle overtaking and distributed passenger boarding behaviour. Transportation Research Part B: Methodological 104, 175-197.

Xuan, Y., Argote, J., Daganzo, C.F., 2011. Dynamic bus holding strategies for ${ }_{730}$ schedule reliability: Optimal linear control and performance analysis. Transportation Research Part B: Methodological 45, 1831-1845.

Yu, H., Chen, D., Wu, Z., Ma, X., Wang, Y., 2016. Headway-based bus bunching prediction using transit smart card data. Transportation Research Part C: Emerging Technologies 72, 45-59. 


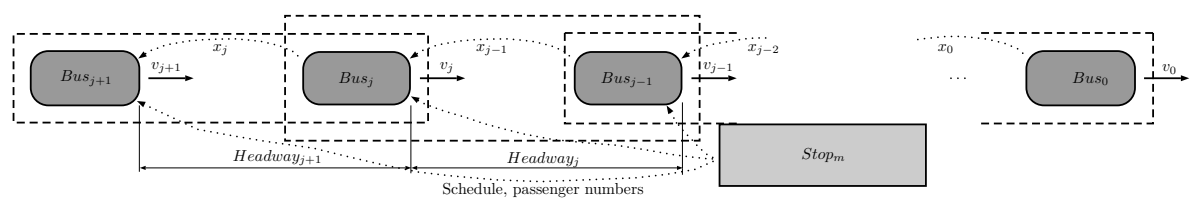

Figure 1: Overlapped, decentralized control strategy

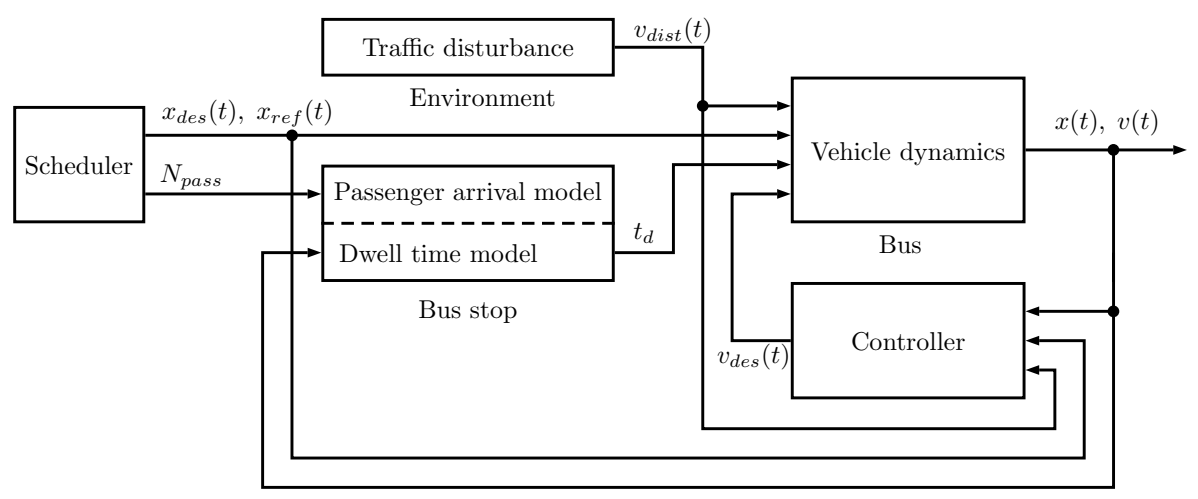

Figure 2: Control system architecture

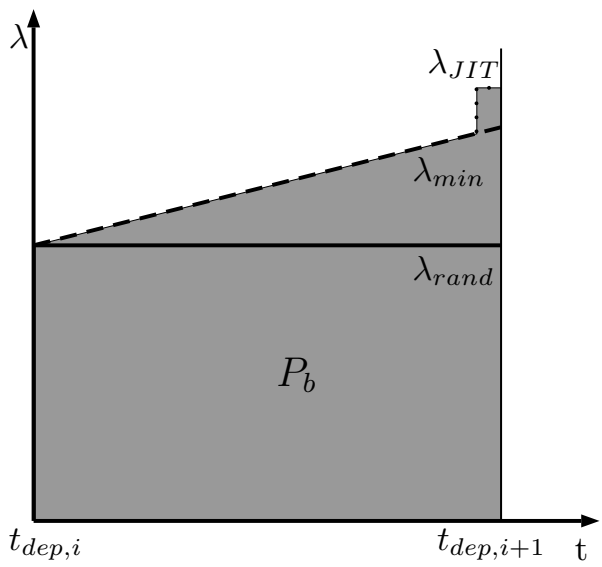

Figure 3: Arrival rate of each passenger type: $a$ ) coincident or just in time (JIT) arrivals $\left.\left(\lambda_{J I T}\right), b\right)$ waiting time minimizers $\left.\left(\lambda_{\min }\right), c\right)$ random arrivals $\left(\lambda_{\text {rand }}\right)$ 


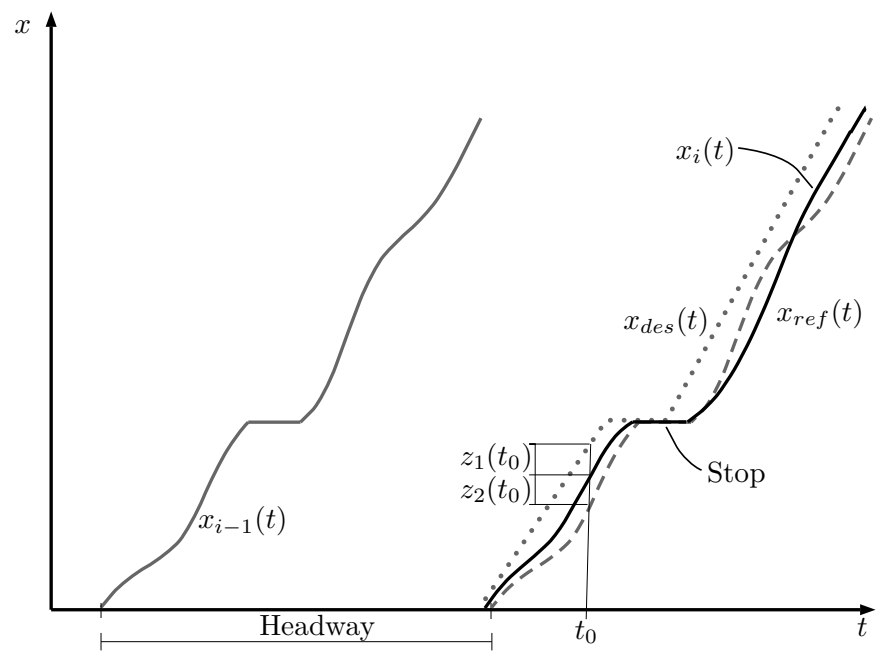

Figure 4: Reference tracking

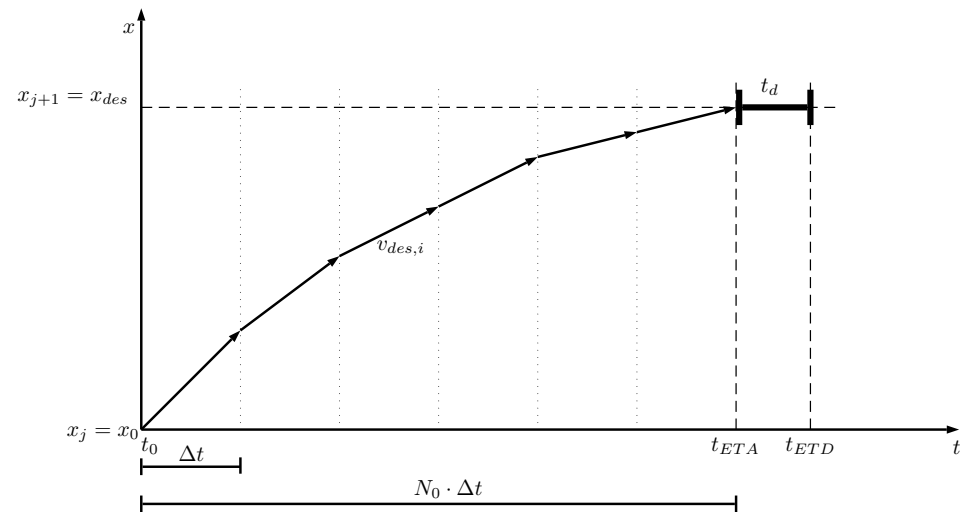

Figure 5: MPC trajectory control 


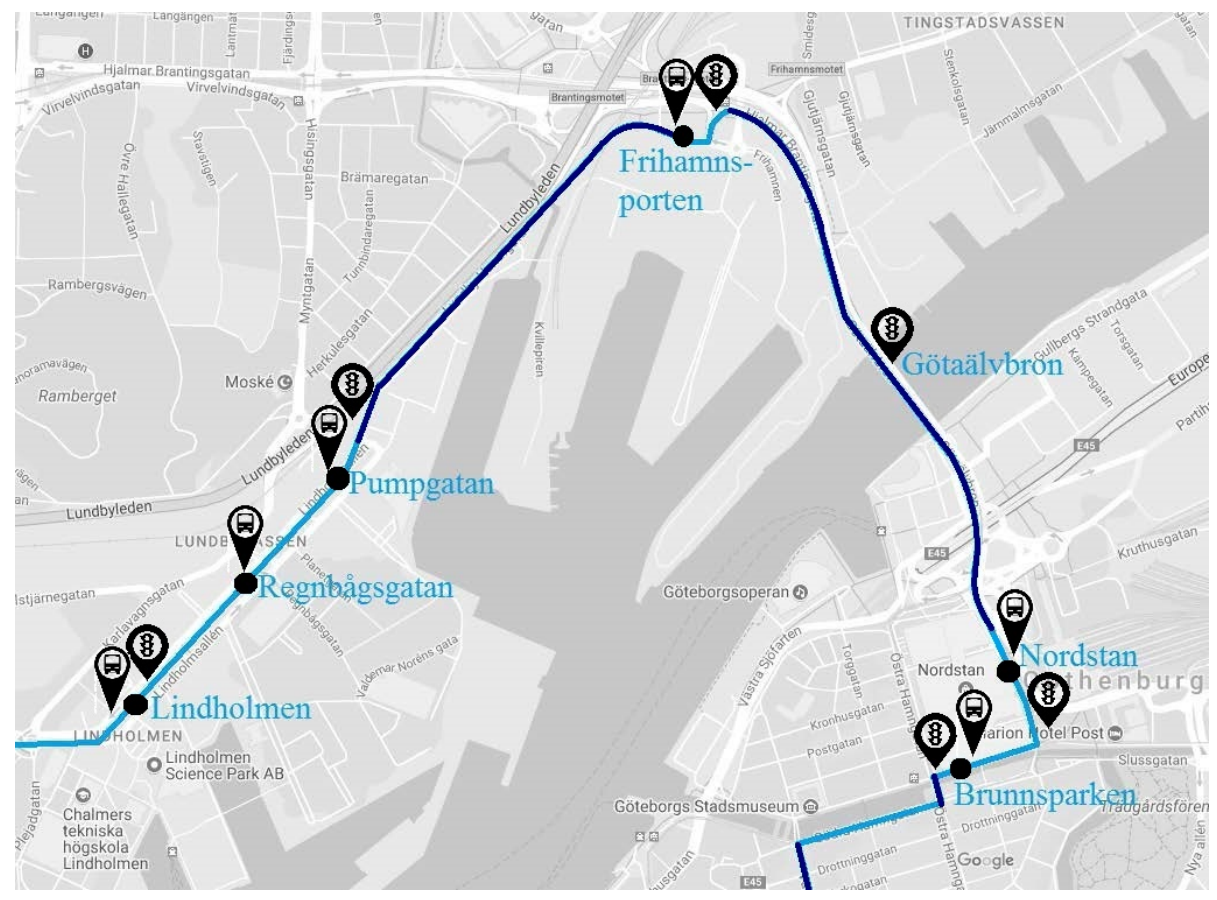

Figure 6: Modeled route section, Gothenburg, Line 16. Dots mark stops and semaphore pictograms indicate traffic lights. The route in darker shade of blue represents mixed traffic (i.e. lack of dedicated bus lane). (GPS coordinates: 57.7109,11.9436;source: Google maps) 


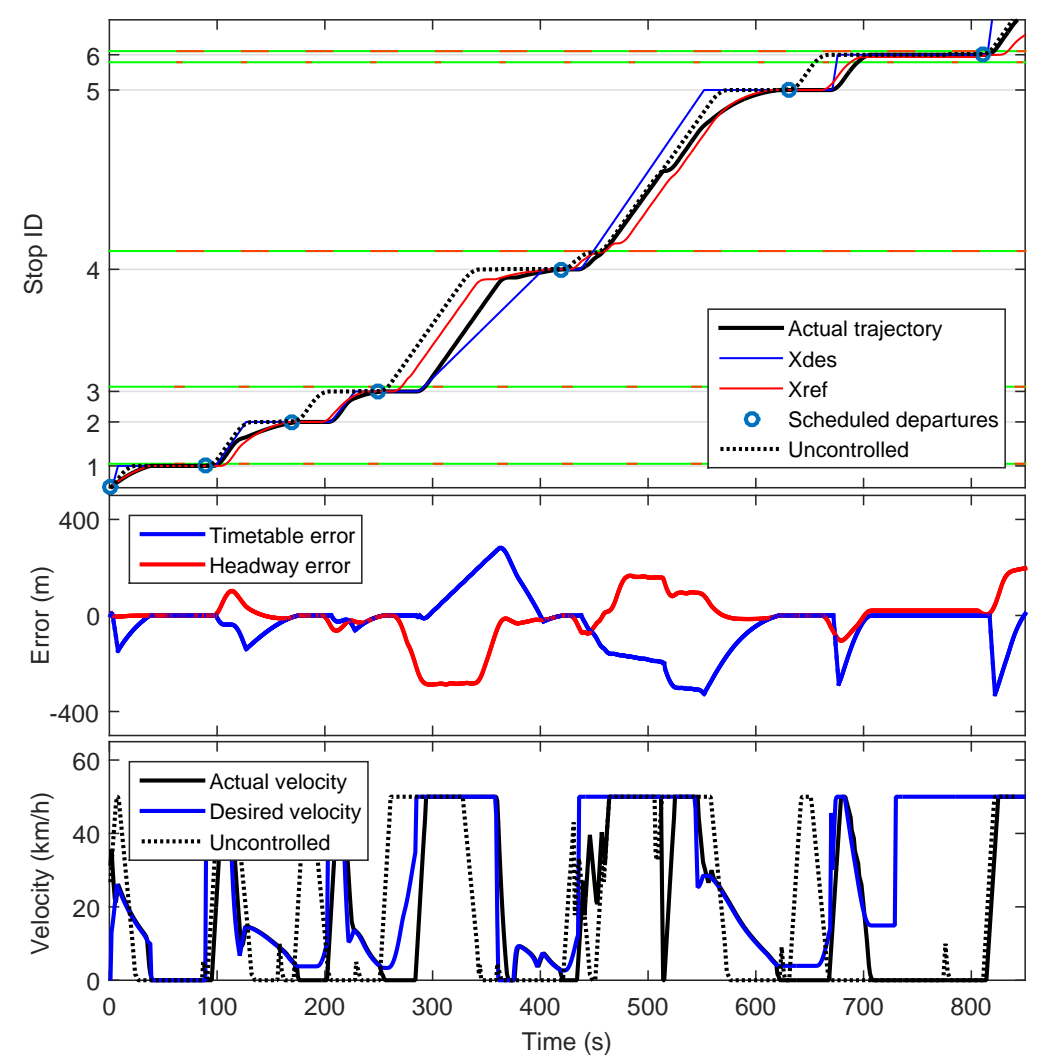

Figure 7: Bus trajectory and velocity profile $\left(5^{\text {th }}\right.$ trajectory in Figure 16 . 

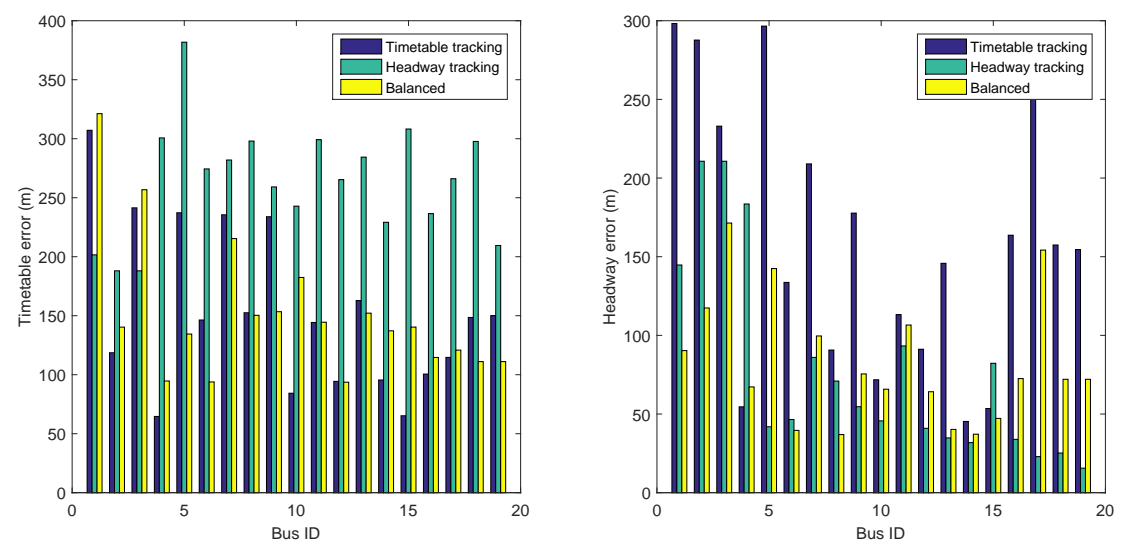

Figure 8: Timetable (left) and Headway (right) errors

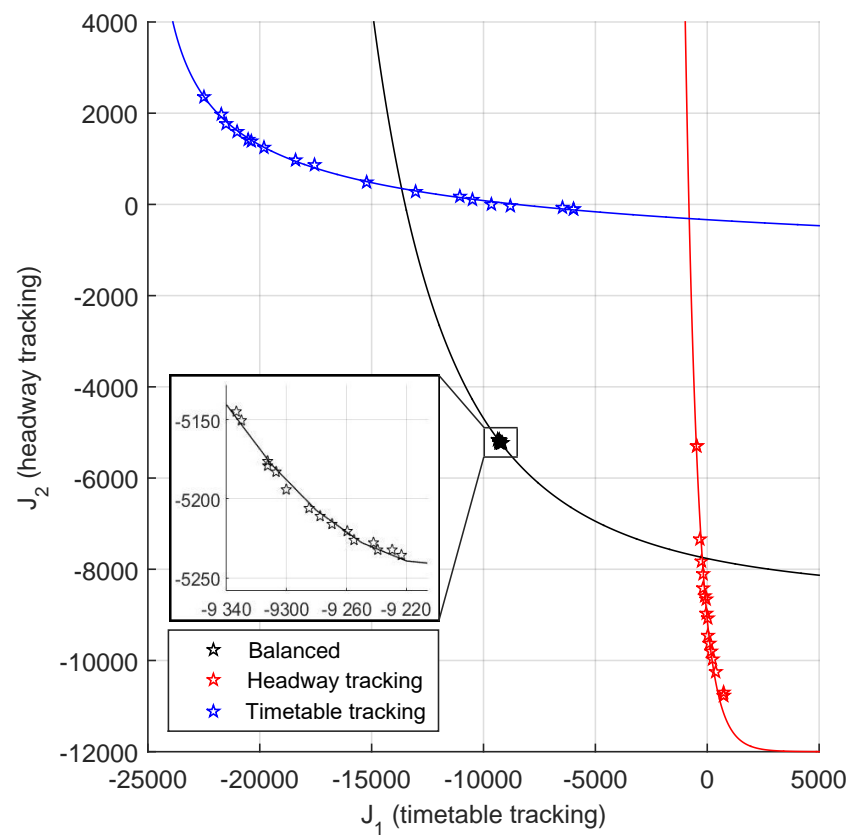

Figure 9: Pareto Front 


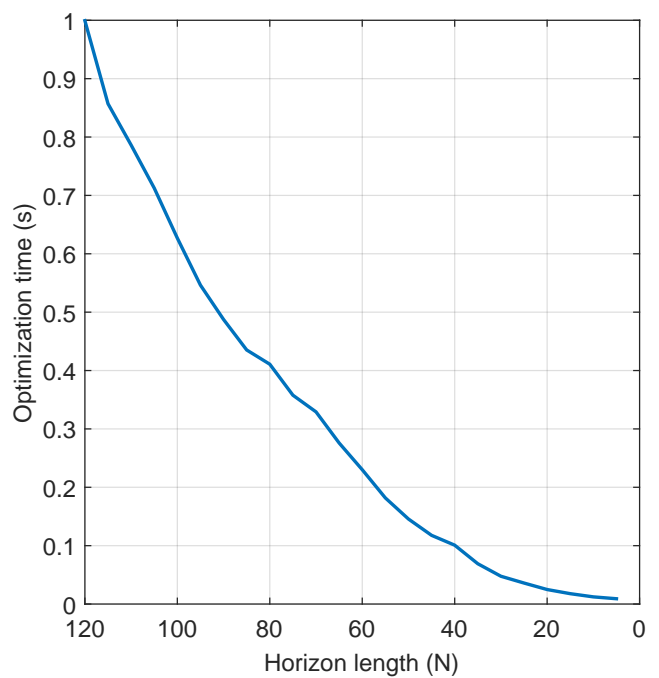

Figure 10: Optimization time 


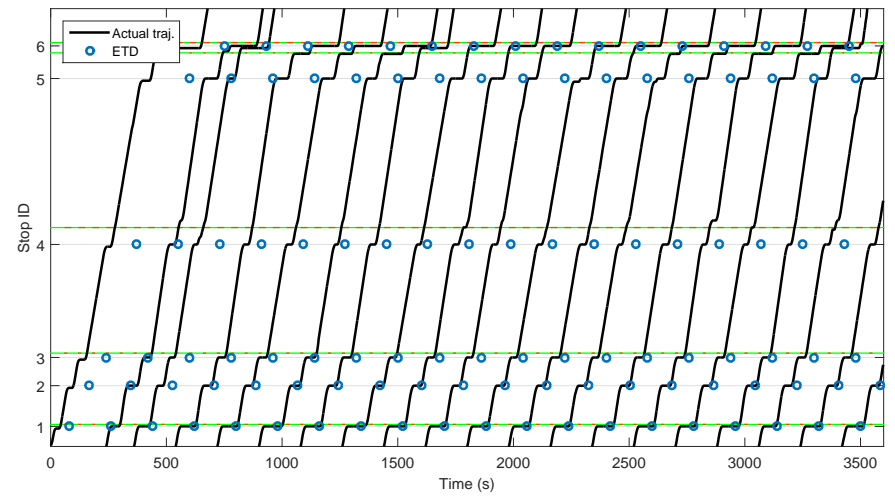

Figure 11: Bus trajectories - uncontrolled

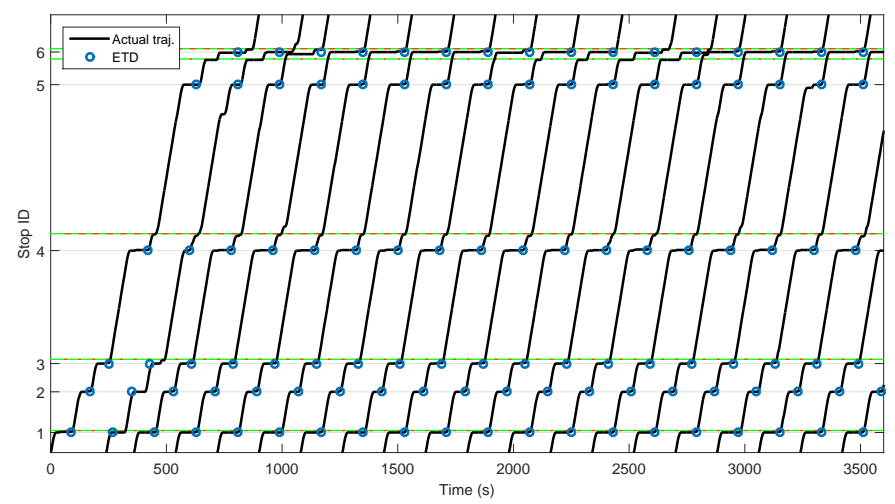

Figure 12: Bus trajectories - holding 


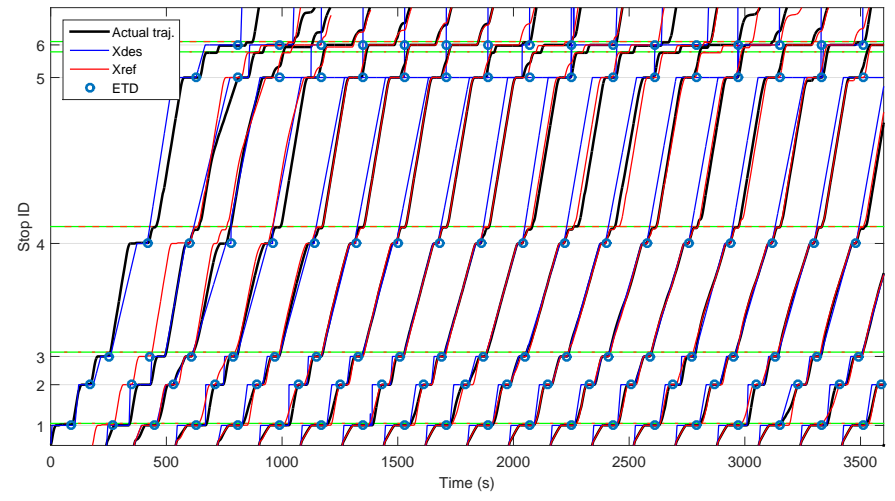

Figure 13: Bus trajectories - PI control

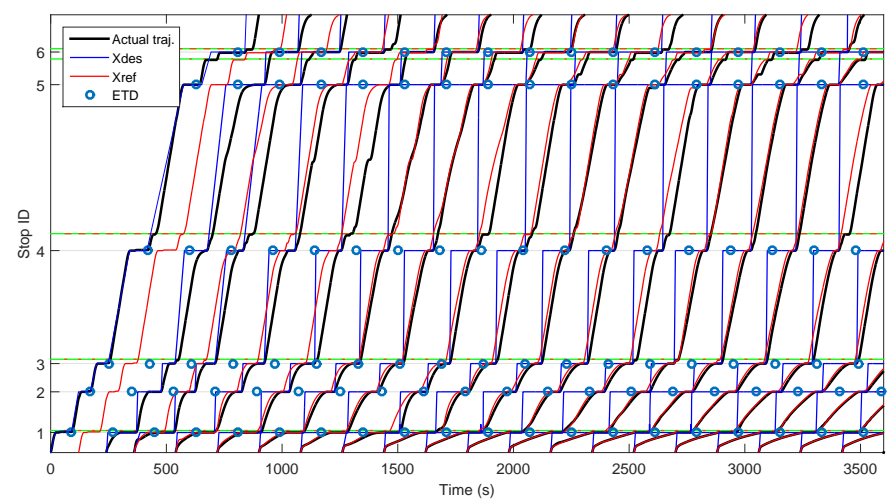

Figure 14: Bus trajectories - headway tracking 


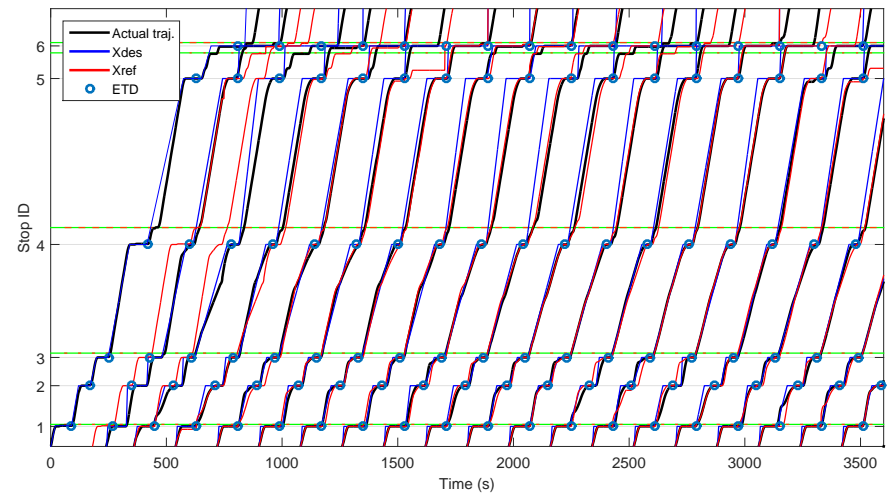

Figure 15: Bus trajectories - timetable tracking

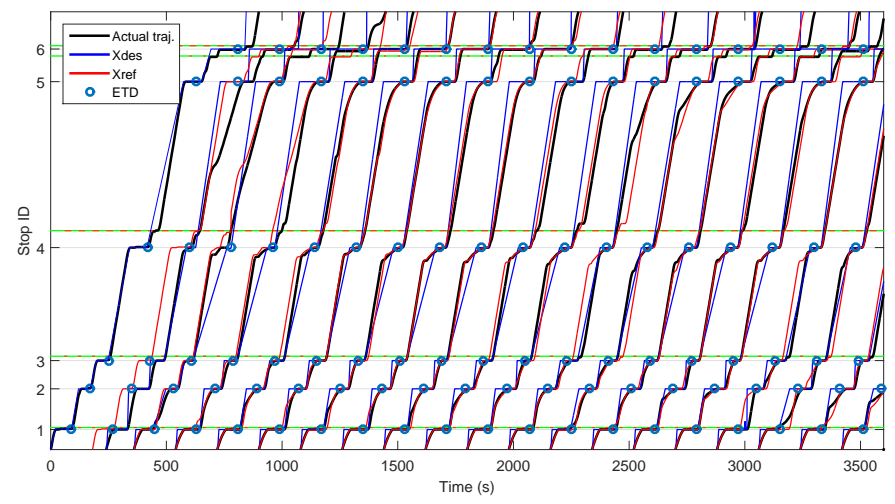

Figure 16: Bus trajectories - balanced control 


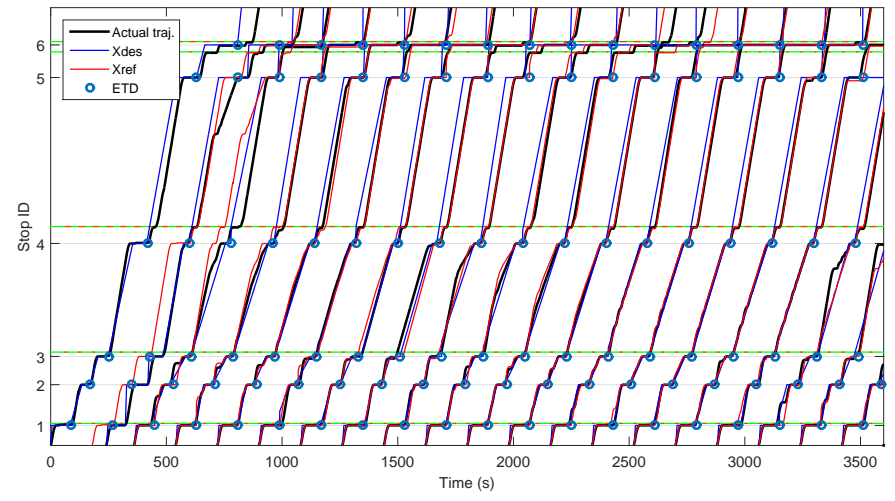

Figure 17: Bus trajectories - adaptive balanced control

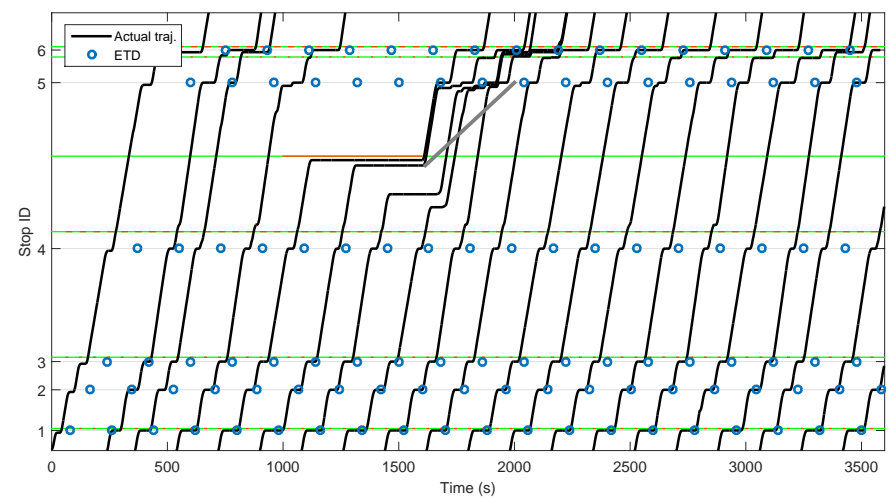

Figure 18: Bus trajectories with service disruption - uncontrolled 


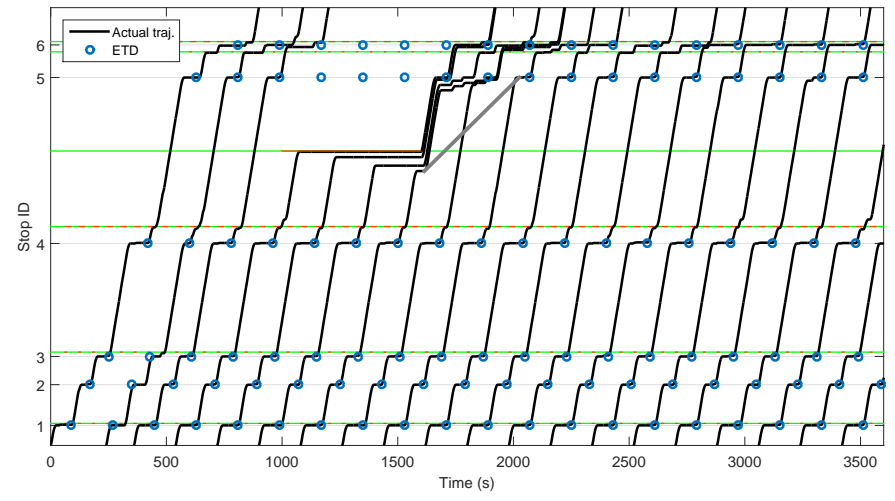

Figure 19: Bus trajectories with service disruption - holding

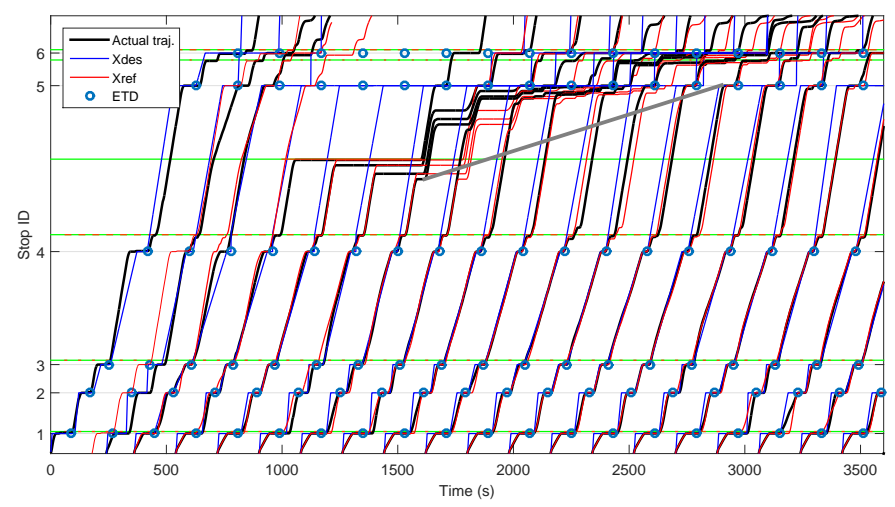

Figure 20: Bus trajectories with service disruption - PI control 


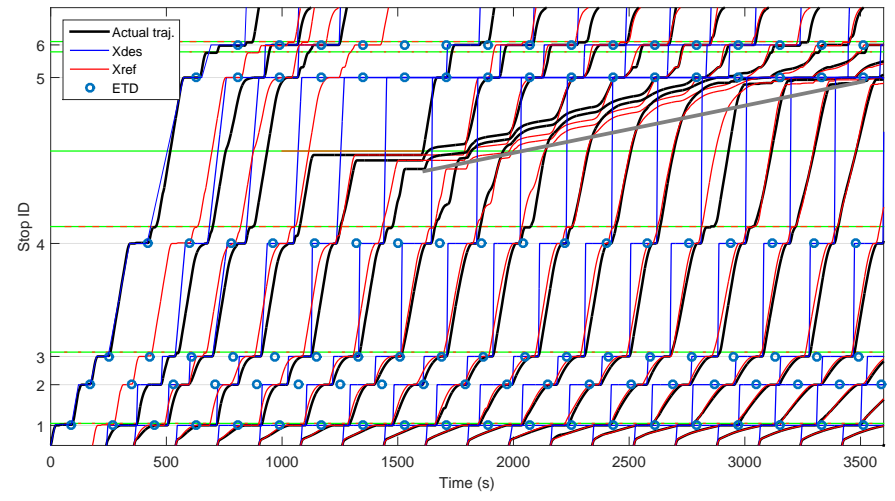

Figure 21: Bus trajectories with service disruption - headway tracking

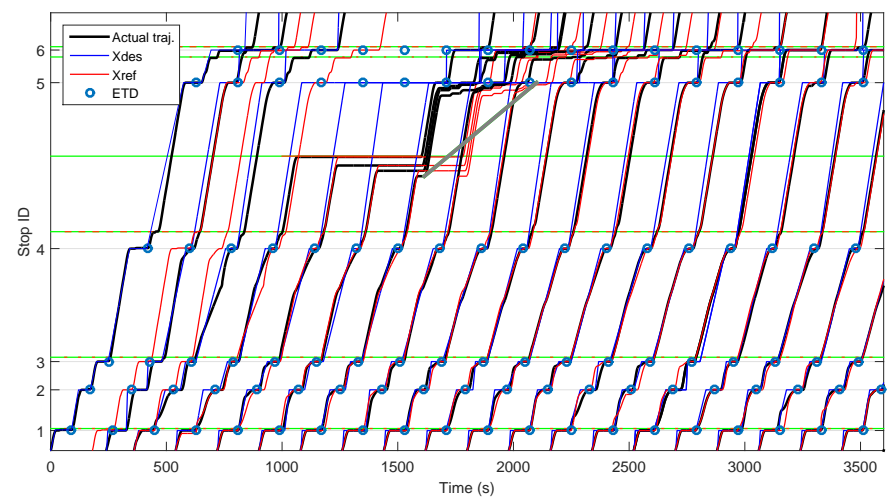

Figure 22: Bus trajectories with service disruption - timetable tracking 


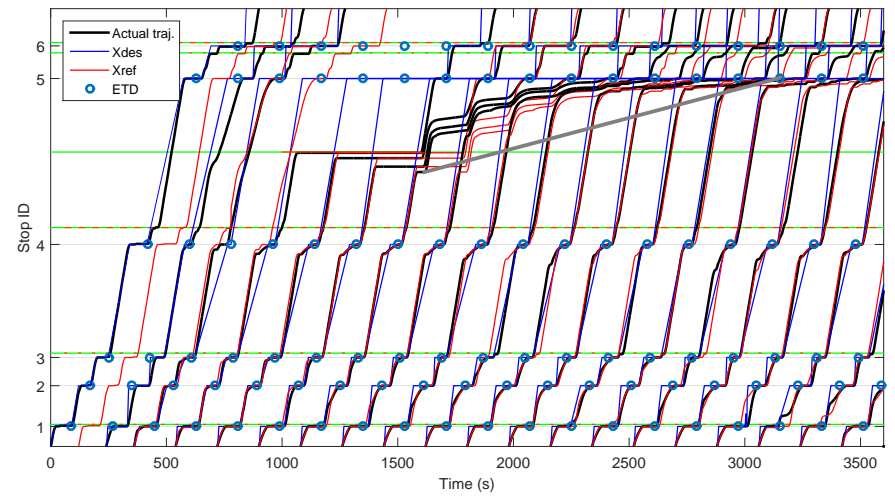

Figure 23: Bus trajectories with service disruption - balanced control

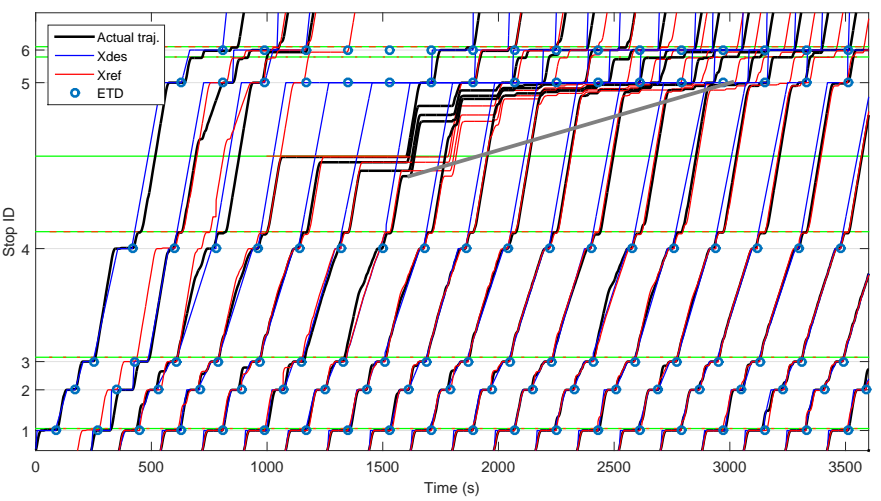

Figure 24: Bus trajectories with service disruption - adaptive balanced control 